\title{
TORIC MODIFICATIONS OF CYCLIC ORBIFOLDS AND AN EXTENDED ZAGIER RECIPROCITY FOR DEDEKIND SUMS
}

\author{
Dedicated to the memory of Professor Masayoshi Nagata
}

TADASHI ASHIKAGA

(Received May 7, 2013, revised April 21, 2014)

\begin{abstract}
We study a toric modification of Fujiki-Oka type for cyclic quotient singularities. Especially the behavior of rational Chow rings, orbifold signatures and so on are explicitly calculated. As a result, we extend Zagier's reciprocity for higher-dimensional Dedekind sums. Namely, we define Dedekind sums with weight by using Atiyah-Singer's equivariant signature with non-isolated fixed point locus, and then prove our reciprocity among them.
\end{abstract}

Introduction. This paper concerns with singularity theory and number theory via the methods of toric geometry. By extending the notion of Hilbert modular cusps, Tsuchihashi [Tsu] defined higher-dimensional cusp singularities via toric geometry. Ogata [Og1] [Og2] studied zeta functions, eta functions and signature defects for these cusp singularities. Ishida [I2] also studied the special value of the zeta functions of these singularities. Our studies are on the same line as the above works in the spirit, but our objects are not cusp singularities but cyclic quotient singularities. We start to explain it from the viewpoint of singularity.

0.1. Let $X$ be an $n$-dimensional analytic space which has as isolated cyclic quotient singularity at the origin $\mathbf{o} \in X$. If $n=2$, the resolution process of the singularity $(X, \mathbf{o})$ is classically well-known via Hirzebruch-Jung method. If $n \geq 3$, Fujiki [Fuj] first constructed a certain resolution process for $(X, \mathbf{o})$, and other types of processes are known in these days.

Oka $[\mathrm{Ok}]$ constructed a certain resolution process for toric singularities. Note that cyclic quotient singularities belong to a class of toric singularities which come from semi-unimodular cones (§1.2). If one applies Oka's subdivision lemma ([Ok, p. 410]) to a semi-umimodular cone, then the resulting toric modification $\mu: Y \rightarrow X$ is nothing but the birational holomorphic map which is used by Fujiki as the key lemma [Fuj, Lemma 3] of his construction. We call $\mu$ Fujiki-Oka modification.

The aim of this paper is to study geometric and number-theoretic properties of FujikiOka modifications. We show the following:

(i) We previously define a natural multi-dimensional Euclidian algorithm (Definition 1.1.1). Then the space $Y$ has cyclic quotient singularities whose types essentially

2010 Mathematics Subject Classification. Primary 11F20; Secondary 14M25, 32S45, 58J20, 11F23, 14B05, 57R18.

Key words and phrases. Singularity, toric geometry, Dedekind sum, reciprocity, signature, orbifold.

Partly supported by the Grant-in-Aid for Scientific Research (C), Japan Society for the Promotion of Science. 
match with this algorithm (Lemma 1.2.5). The singularities of $Y$ are no more isolated in general.

(ii) The morphism $\mu$ has a relative projective orbifold compactification $\bar{\mu}: \bar{Y} \rightarrow \bar{X}$ (Proposition 1.3.1), and the relative Chow ring for $\bar{\mu}$ is described (Remark 3.2.2).

Now we assume that $n$ is even, and study the behavior via $\bar{\mu}$ of invariants related to signatures. For even dimensional complex orbifold $M$ in general, two types of signatures are defined. The one is the (usual) signature $\operatorname{Sign} M$ as a rationally homology manifold, and the other is the orbifold signature $\operatorname{Sign}^{\text {orb }} M$ which is defined by the integral of orbifold L-class. The difference $\operatorname{Sdef} M:=\operatorname{Sign} M-\operatorname{Sign}^{\text {orb }} M$ is called $S$-defect ${ }^{1}$, which is essentially described by Lefschetz fixed point formula for the signature operator ([AS], [Ka]). We calculate the relative contributions of $\bar{\mu}$ to these three invariants.

(iii) The difference of signatures $\operatorname{Sign} \bar{Y}-\operatorname{Sign} \bar{X}$ is calculated by using Leung-Reiner's theorem [LR] (Corollary 2.1.2).

(iv) The difference of orbifold signatures $\operatorname{Sign} \bar{Y}^{\text {orb }}-\operatorname{Sign}^{\text {orb }} \bar{X}$ is calculated by using the method of Pommersheim [P] (Proposition 3.2.1).

(v) The difference of S-defects $\operatorname{Sdef} \bar{Y}-\operatorname{Sdef} \bar{X}$ is calculated according to Kawasaki's formula [Ka] (Proposition 5.1.2).

By substituting the results of (iii)-(v) to the equality

$$
(\operatorname{Sign} \bar{Y}-\operatorname{Sign} \bar{X})-\left(\operatorname{Sign}^{\text {orb }} \bar{Y}-\operatorname{Sign}^{\text {orb }} \bar{X}\right)=\operatorname{Sdef} \bar{Y}-\operatorname{Sdef} \bar{X},
$$

we have our reciprocity formula for generalized Dedekind sums (Theorem 4.2.2). We explain a number-theoretic meaning of it in the next subsection.

0.2. Historically, in order to express the explicit $S L_{2}(\mathbf{Z})$ actions to his eta function, Dedekind introduced the notion of Dedekind sums

$$
s(a ; d)=\sum_{k=1}^{d-1}\left(\left(\frac{k}{d}\right)\right)\left(\left(\frac{k a}{d}\right)\right)
$$

where $d, a$ are mutually prime natural numbers and $((x))=x-[x]-1 / 2(x \notin \mathbf{Z}),((x))=0$ $(x \in \mathbf{Z})$. He also proved the 2-dimensional inhomogeneous reciprocity

$$
s(a ; d)+s(d ; a)=-\frac{1}{4}+\frac{1}{12}\left(\frac{a}{d}+\frac{a}{d}+\frac{1}{d a}\right) .
$$

Rademacher extended (2) to the 2-dimensional homogeneous reciprocity for the homogeneous Dedekind sum $s\left(a_{1}, a_{2} ; d\right)=\sum_{k=1}^{d-1}\left(\left(k a_{1} / d\right)\right)\left(\left(k a_{2} / d\right)\right)$. He expressed it as

$$
s\left(a_{1}, a_{2} ; d\right)=\frac{1}{4 d} \sum_{k=1}^{d-1} \cot \frac{\pi a_{1} k}{d} \cot \frac{\pi a_{2} k}{d}
$$

\footnotetext{
${ }^{1}$ We may call it signature defect. Although this word is sometimes used in more delicate sense assuming a framing of the tangent bundle, we avoid it here.
} 
by using Fourier expansion (cf. [RG]). As an extended form of (3), Hirzebruch-Zagier [HZ] and Zagier [Z] introduced the notion of higher-dimensional Dedekind sums

$$
s\left(a_{1}, \ldots, a_{n} ; d\right)=\frac{(-1)^{n / 2}}{d} \sum_{k=1}^{d-1} \prod_{i=1}^{n} \cot \left(\frac{\pi k a_{i}}{d}\right)
$$

for an even natural number $n$. Zagier [Z] also proved the $n$-dimensional homogeneous reciprocity

$$
\sum_{i=0}^{n} s\left(a_{0}, \ldots, a_{i-1}, a_{i+1}, \ldots, a_{n} ; a_{i}\right)=1-\frac{L_{n}\left(a_{0}, \ldots, a_{n}\right)}{\prod_{i=0}^{n} a_{i}},
$$

where $a_{0}, a_{1}, \ldots, a_{n}$ are pairwise prime natural numbers and $L_{n}$ is Hirzebruch's L-polynomial of degree $n$.

Beck [B] extended the notion of Dedekind sums which relates to higher derivatives of the cotangent function, and the reciprocity was studied by [B] and [BR]. See for instance [GS], [BH1], [BH2] for other number theoretic related topics.

From the topological viewpoint, the invariant (4) is directly related to the G-signature theorem with an isolated fixed point, i.e., the Lefschetz fixed point formula ([AB, p. 473]) for the signature operator. For the reciprocity related to the Lefschetz fixed point formula of other Dirac operators, see [FFU], [Fuk] etc.

From the algebro-geometric viewpoint, Pommersheim [P] gave an interesting proof for the 2-dimesional homogenenous reciprocity by using toric geometry. Reid [R] defined another type of higher-dimensional Dedekind sum which relates to orbifold Riemann-Roch problem, and this direction was developed by [BRZ]. See for instance [U], [A1] for other related topics.

Now our reciprocity is as follows. We drop the symmetry condition in (5) to the effect that $a_{0}, a_{1}, \ldots, a_{n}$ are pairwise prime, and prove a reciprocity similar to (5). Geometrically, this situation naturally leads us to using the G-signature theorem (cf. [AS], [HZ]) for nonisolated fixed point locus. Our formulation is the following.

We first define the notion of Dedekind sums with weight as follows. By substituting the variables for the data of the normal bundle of the fixed point locus of equivariant L-class, we first define the Atiyah-Singer cotangent function. This is a meromorphic function whose constant term coincides with the usual cotangent product and whose other coefficients are written in terms of higher derivatives of usual cotangent functions and the Bernoulli numbers as in (35). Then the Dedekind sum with weight is defined by substituting the Atiyah-Singer cotangent function for the cotangent function in (4).

We define the notion of $n$-dimensional proper fraction $\mathbf{a} / d=\left(a_{1}, \ldots, a_{n}\right) / d\left(0 \leq a_{i} \leq\right.$ $d-1$ ) and higher-dimensional analogs of the classical Euclidean algorithm in the set of these proper fractions, as mentioned in $\S 0.1$. We call them $i$-th remainder maps and $i$-th round down maps for $1 \leq i \leq n$.

Then our reciprocity (37) is stated as a relation among the remainder maps of the proper fraction $\mathbf{a} / d$ with respect to the Dedekind sums with weight under the assumption that 
$\operatorname{gcd}\left(d, a_{i}\right)=1(1 \leq i \leq n-1)$ and $a_{n}=1$. If we further assume that $a_{1}, \ldots, a_{n-1}$ are pairwise prime, then our reciprocity coincides with inhomogeneous Zagier reciprocity (Corollary 4.2.3). In this sense, our reciprocity is a generalization of Zagier reciprocity.

Lastly we note that our trials are on the first stage and many related problems are open, and some of them are commented in Remark 5.5.1.

1. Fujiki-Oka modification. We express a cyclic orbipoint (a cyclic quotient singularity) as the toric variety corresponding to a semi-unimodular simplicial cone, and define a toric modification via Oka's subdivision of this cone. The algorithm for this subdivision essentially coincides with the Euclidean algorithm for a higher-dimensional proper fraction defined in $\$ 1.1$.

1.1. Let $\mathbf{N}$ and $\mathbf{Z}$ be the set of natural numbers and integers, respectively. We call

$$
\frac{\mathbf{a}}{d}=\frac{\left(a_{1}, \ldots, a_{n}\right)}{d}
$$

an $n$-dimensional proper fraction $(n \geq 1)$ if $d \in \mathbf{N}$ and $\mathbf{a}=\left(a_{1}, \ldots, a_{n}\right) \in \mathbf{Z}^{n}$ satisfy $0 \leq a_{i} \leq d-1$ for $1 \leq i \leq n$. Here we call $\mathbf{a}$ and $d$ the numerator and the denominator of $\mathbf{a} / d$, respectively, and call $a_{i}$ the $i$-th component of the numerator. The prototype of this notion comes from Reid [R].

Let $\mathbf{Q}_{n}^{\text {prop }}$ be the set of $n$-dimensional proper fractions, and let $\overline{\mathbf{Q}}_{n}^{\text {prop }}$ be the set of union of $\mathbf{Q}_{n}^{\text {prop }}$ and the symbol $\infty$. Similarly, we set $\overline{\mathbf{Z}}^{n}=\mathbf{Z}^{n} \cup\{\infty\}$. If the $n$-th component of the numerator satisfies $a_{n}=1$, then $\mathbf{a} / d$ is said to be inhomogeneous. If $\mathbf{a} / d \in \mathbf{Q}_{n}^{\text {prop }}$ satisfies

$$
\operatorname{gcd}\left(d, a_{i}\right)=1 \quad(1 \leq i \leq n),
$$

then it is said to be irreducible. Moreover an irreducible proper fraction $\mathbf{a} / d$ satisfying

$$
\operatorname{gcd}\left(a_{i}, a_{j}\right)=1 \quad(1 \leq i<j \leq n)
$$

is said to be absolutely irreducible. The multiple of $\mathbf{a} / d$ by $k \in \mathbf{Z}$ is defined by

$$
k \cdot \frac{\mathbf{a}}{d}=\frac{\left(\overline{k a_{1}}, \ldots, \overline{k a_{n}}\right)}{d} \in \mathbf{Q}_{n}^{\text {prop }}
$$

where $\overline{k a_{i}} \equiv k a_{i}(\bmod d)$ with $0 \leq \overline{k a_{i}} \leq d-1$ (cf. [R, p. 372]). We sometimes write the numerator of $\mathbf{a} / d$ by $\mathbf{a}=\operatorname{Num}(\mathbf{a} / d)$. On the other hand, the $i$-th shifted numerator of $\mathbf{a} / d$ is defined by

$$
N_{i}\left(\frac{\mathbf{a}}{d}\right)=\left(a_{1}, \ldots, a_{i-1},-d, a_{i+1}, \ldots, a_{n}\right) \in \mathbf{Z}^{n} .
$$

Now we define an analog for $\mathbf{Q}_{n}^{\text {prop }}$ of the classical Euclidean algorithm as follows:

DeFinition 1.1.1. (i) For $1 \leq i \leq n$, the $i$-th round down map $Z_{i}: \overline{\mathbf{Q}}_{n}^{\text {prop }} \rightarrow \overline{\mathbf{Z}}^{n}$ is defined by

$Z_{i}\left(\frac{\left(a_{1}, \ldots, a_{n}\right)}{d}\right)= \begin{cases}\left(\left\lfloor\frac{a_{1}}{a_{i}}\right\rfloor, \ldots,\left\lfloor\frac{a_{i-1}}{a_{i}}\right\rfloor,\left\lfloor\frac{-d}{a_{i}}\right\rfloor,\left\lfloor\frac{a_{i+1}}{a_{i}}\right\rfloor, \ldots,\left\lfloor\frac{a_{n}}{a_{i}}\right\rfloor\right) & \text { if } a_{i} \neq 0 \\ \infty & \text { if } a_{i}=0\end{cases}$ 
and $Z_{i}(\infty)=\infty$. Here $\lfloor x\rfloor$ is the greatest integer not exceeding $x$.

(ii) The $i$-th remainder map $R_{i}: \overline{\mathbf{Q}}_{n}^{\text {prop }} \rightarrow \overline{\mathbf{Q}}_{n}^{\text {prop }}$ is defined by

$$
R_{i}\left(\frac{\left(a_{1}, \ldots, a_{n}\right)}{d}\right)= \begin{cases}\frac{\left(\overline{a_{1}}, \ldots, \overline{a_{i-1}}, \overline{(-d)}, \overline{a_{i+1}}, \ldots, \overline{a_{n}}\right)}{a_{i}} & \text { if } a_{i} \neq 0 \\ \infty & \text { if } a_{i}=0\end{cases}
$$

and $R_{i}(\infty)=\infty$.

For instance, if $\alpha=(2,6,3,1) / 7$, then $Z_{3}(\alpha)=(0,2,-3,0)$ and $R_{3}(\alpha)=(2,0,2,1) /$ 3.

In the usual addition on $\mathbf{Z}^{n}$, we have

$$
\operatorname{Num}\left(R_{i}(\mathbf{a} / d)\right)+a_{i} Z_{i}(\mathbf{a} / d)=N_{i}(\mathbf{a} / d), \quad \text { if } a_{i} \neq 0 .
$$

Applications of the iteration of this algorithm are discussed in [A2].

1.2. We fix an inhomogeneous proper fraction $\mathbf{a} / d=\left(a_{1}, \ldots, a_{n-1}, 1\right) / d \in \mathbf{Q}_{n}^{\text {prop }}$ and a primitive $d$-th root of unity $\xi$. Let $\left(z_{1}, \ldots, z_{n}\right)$ be a complex coordinate of $\mathbf{C}^{n}$ and let $\varphi_{\mathbf{a} / d}: \mathbf{C}^{n} \rightarrow \mathbf{C}^{n}$ be the holomorphic automorphism defined by

$$
\varphi_{\mathbf{a} / d}:\left(z_{1}, \ldots, z_{n-1}, z_{n}\right) \longmapsto\left(\xi^{a_{1}} z_{1}, \ldots, \xi^{a_{n-1}} z_{n-1}, \xi z_{n}\right) .
$$

The germ at the origin of the quotient space $\mathbf{C}^{n} /\left\langle\phi_{\mathbf{a} / d}\right\rangle$ with respect to the cyclic group $\left\langle\phi_{\mathbf{a} / d}\right\rangle$ generated by $\phi_{\mathbf{a} / d}$ is called a cyclic orbipoint of type $\mathbf{a} / d$. If $\mathbf{a} / d$ is an irreducible fraction, then this singularity is isolated. In general, it need not be isolated.

Now we interpret this quotient space in terms of toric geometry. We follow Oda [Od] for the basic terminology of toric geometry. We fix a free abelian group $N \simeq \mathbf{Z}^{n}$ of rank $n$. We put $M=\operatorname{Hom}_{\mathbf{Z}}(N, \mathbf{Z})$ and denote by

$$
\langle\rangle: M \times N \longrightarrow \mathbf{Z}
$$

the canonical pairing. We put $N_{\mathbf{R}}=N \otimes_{\mathbf{Z}} \mathbf{R}, M_{\mathbf{R}}=M \otimes_{\mathbf{Z}} \mathbf{R}$, and also denote by \langle\rangle : $M_{\mathbf{R}} \times N_{\mathbf{R}} \rightarrow \mathbf{R}$ the natural pairing. Let $P_{i}={ }^{t}\left(P_{i 1}, \ldots, P_{i n}\right) \in N$ be primitive elements for $1 \leq i \leq n$, and let

$$
\sigma=\mathbf{R}_{\geq 0} P_{1}+\cdots+\mathbf{R}_{\geq 0} P_{n}
$$

be the $n$-dimensional simplicial cone generated by $P_{1}, \ldots, P_{n}$. Let $\triangle_{\sigma}$ be the fan consisting of all the faces of $\sigma$, and let $U_{\sigma}$ be the affine toric variety corresponding to $\triangle_{\sigma}$. Namely $U_{\sigma}$ is defined to be the space of semi-group homomorphisms $\operatorname{Hom}_{\text {semigr }}(\mathbf{C}[\check{\sigma} \cap M], \mathbf{C})$ sending 0 to 0 , where $\check{\sigma}=\left\{x \in M_{\mathbf{R}} ;\langle x, y\rangle \geq 0, y \in \sigma\right\}$ is the dual cone of $\sigma$.

For an $m$-dimensional face $\tau=\mathbf{R}_{\geq 0} P_{i_{1}}+\cdots+\mathbf{R}_{\geq 0} P_{i_{m}}$ of $\sigma$ (for $m \leq n$ ), the greatest common divisor of the absolute values of the $m \times m$ minors of the matrix $\left\{p_{i_{k} j}\right\}_{1 \leq k \leq m, 1 \leq j \leq n}$ is called the multiplicity of $\tau$, and is denoted by mult $\tau$. Note that mult $\tau=1$ if and only if $P_{i_{1}}, \ldots, P_{i_{m}}$ forms a part of a $\mathbf{Z}$-basis of $N$. If mult $\sigma=1$, then $\sigma$ is said to be nonsingular 
(or unimodular). In this case, the variety $U_{\sigma}$ is non-singular. As the simplest simplicial cone but singular, we define the following:

DEFINITION 1.2.1. A simplicial cone $\sigma$ as in (10) is said to be semi-unimodular over $P_{n}$ if the multiplicity of the face $\mathbf{R}_{\geq 0} P_{1}+\cdots+\mathbf{R}_{\geq 0} P_{n-1}$ is one, i.e., the set of primitive vectors $\left\{P_{1}, \ldots, P_{n-1}\right\}$ is a part of $\mathbf{Z}$-basis of $N$. In this case, $P_{n}$ is called the exceptional element.

The above notion intrinsically appear in Oka [Ok], where an inductive method for a general toric resolution was studied. The following proposition is due to Oka.

Poposition 1.2.2 ([Ok, p. 410]). Suppose $\sigma=\mathbf{R}_{\geq 0} P_{1}+\cdots+\mathbf{R}_{\geq 0} P_{n}$ is semi-unimodular over $P_{n}$ with mult $\sigma=d>1$. Let $Q \in N$ be a primitive element such that

(a) The ray $\mathbf{R}_{\geq 0} Q$ is contained in $\sigma$, i.e., $Q$ is written as $\sum_{i=1}^{n} r_{i} P_{i}$ for $r_{i} \in \mathbf{R}_{\geq 0}$.

(b) The cone $\mathbf{R}_{\geq 0} P_{1}+\cdots+\mathbf{R}_{\geq 0} P_{n-1}+\mathbf{R}_{\geq 0} Q$ is non-singular.

Then the following (i) and (ii) hold:

(i) There exist non-negative integers $a_{1}, \ldots, a_{n-1}$ such that $Q$ is written as

$$
Q=\frac{\sum_{i=1}^{n-1} a_{i} P_{i}+P_{n}}{d}
$$

(ii) The integers $a_{1}, \ldots, a_{n-1}$ in (11) are unique modulo $d$. Especially there exists a unique element $Q \in N$ which satisfies (11) under the assumption $0 \leq a_{i} \leq d-1$.

By Proposition 1.2.2, (ii), the inhomogeneous proper fraction $\mathbf{a} / d=\left(a_{1}, \ldots, a_{n-1}, 1\right) / d$ is uniquely determined for a given semi-unimodular cone $\sigma$ over $P_{n}$, which we call the proper fraction of $\sigma$. The primitive element $Q \in N$ in (11) determined by the proper fraction of $\sigma$ is called Oka center of $\sigma$.

PROPOSITION 1.2.3. Let $\sigma=\mathbf{R}_{\geq 0} P_{1}+\cdots+\mathbf{R}_{\geq 0} P_{n}$ be semi-unimodular over $P_{n}$ with the proper fraction $\mathbf{a} / d$. Then the germ at the origin of the toric variety $U_{\sigma}$ coincides with the cyclic orbipoint of type $\mathbf{a} / d$.

Proof. Let $Q$ be Oka center of $\sigma$. Since the simplex $\mathbf{R}_{\geq 0} P_{1}+\cdots+\mathbf{R}_{\geq 0} P_{n-1}+\mathbf{R}_{\geq 0} Q$ is non-singular, $\left\{P_{1}, \ldots, P_{n-1}, Q\right\}$ forms a $\mathbf{Z}$-basis of $N$. We put $P_{n}=\sum_{i=1}^{n-1} x_{i} P_{i}+x^{\prime} Q$ for $x_{i}, x^{\prime} \in \mathbf{Z}$. Then

$$
d=\left|\operatorname{det}\left(P_{1}, \ldots, P_{n-1}, \sum_{i=1}^{n-1} x_{i} P_{i}+x^{\prime} Q\right)\right|=x^{\prime}\left|\operatorname{det}\left(P_{1}, \ldots, P_{n-1}, Q\right)\right|=x^{\prime} .
$$

Therefore the expression $Q=\left(-\sum_{i=1}^{n-1} x_{i} P_{i}+P_{n}\right) / d$ implies that $x_{i}=-a_{i}$. Then we have

$$
P_{n}=-\sum_{i=1}^{n-1} a_{i} P_{i}+d Q \text {. }
$$

Let $N^{\prime}$ be the $\mathbf{Z}$-submodule of $N$ of index $d$ generated by $P_{1}, \ldots, P_{n-1}, P_{n}$. Let $\left\{m_{1}, \ldots, m_{n}\right\}$ be the dual $\mathbf{Z}$-basis of $M$ with respect to $\left\{P_{1}, \ldots, P_{n-1}, Q\right\}$, and let $\left\{m_{1}^{\prime}, \ldots, m_{n}^{\prime}\right\}$ be the dual $\mathbf{Z}$-basis of $M^{\prime}=\operatorname{Hom}_{\mathbf{Z}}\left(N^{\prime}, \mathbf{Z}\right)$ with respect to $\left\{P_{1}, \ldots, P_{n-1}, P_{n}\right\}$. By easy calculation using 
(12), we have

$$
m_{i}=m_{i}^{\prime}-a_{i} m_{n}^{\prime} \quad(1 \leq i \leq n-1), \quad m_{n}=d m_{n}^{\prime} .
$$

We identify $N_{\mathbf{R}}^{\prime}=N^{\prime} \otimes_{\mathbf{Z}} \mathbf{R}$ with $N_{\mathbf{R}}$, and let $\left(N^{\prime}, \Delta_{\sigma}\right) \rightarrow\left(N, \Delta_{\sigma}\right)$ be the map of fans of the identical $\Delta_{\sigma}$ with respect to the mutually dinstinct lattices $N^{\prime}$ and $N$. Let

$$
U_{\sigma}^{\prime}=\mathrm{T}_{\mathrm{N}^{\prime}} \mathrm{emb}\left(\Delta_{\sigma}\right) \longrightarrow U_{\sigma}=\mathrm{T}_{\mathrm{N}} \mathrm{emb}\left(\Delta_{\sigma}\right)
$$

be the natural equivariant morphism. Then $U_{\sigma}^{\prime} \simeq \mathbf{C}^{n}$ and (14) is the quotient map by the kernel of the map $T_{N^{\prime}} \rightarrow T_{N}$, which is the cyclic group of order $d$. By the same argument as in the proof of [Od, Prop.1.24], it follows from (13) that (14) is nothing but the quotient map by the action (9).

REMARK 1.2.4. The notions of the proper fraction and Oka center for a semi-unimodular cone depend on the exceptional element. Namely the cone $\sigma$ as above may possibly be semi-unimodular over another $P_{k}$ for $k \neq n$. Then it may have another proper fraction and another Oka center if one changes the exceptional element. In the viewpoint of Proposition 1.2.3, the choice of the exceptional element of a fixed semi-unimodular cone relates to the change of the primitive $d$-th root of unity of the action (9). In fact, if $\xi^{a_{k}}$ in (9) is another primitive $d$-th root of unity for some $k$, then $\sigma$ is also semi-unimodular over $P_{k}$.

LEMMA 1.2.5. Let $\sigma=\mathbf{R}_{\geq 0} P_{1}+\cdots+\mathbf{R}_{\geq 0} P_{n}$ be semi-unimodular over $P_{n}$ whose properfraction is $\mathbf{a} / d=\left(a_{1}, \ldots, a_{n-1}, 1\right) / d$ with Oka center $Q$. If $a_{i} \neq 0$, then the simplicial cone

$$
\sigma_{i}=\mathbf{R}_{\geq 0} P_{1}+\cdots+\mathbf{R}_{\geq 0} P_{i-1}+\mathbf{R}_{\geq 0} Q+\mathbf{R}_{\geq 0} P_{i+1}+\cdots+\mathbf{R}_{\geq 0} P_{n}
$$

is semi-unimodular over $P_{n}$ with multiplicity $a_{i}$. Oka center $Q_{i}$ of $\sigma_{i}$ coincides with

$$
Q_{i}=\frac{\left.\sum_{j=1}^{i-1} \overline{a_{j}} P_{j}+\overline{(-d}\right) Q+\sum_{j=i+1}^{n-1} \overline{a_{j}} P_{j}+P_{n}}{a_{i}},
$$

where $\overline{a_{j}} \equiv a_{j}\left(\bmod a_{j}\right)$ and $0 \leq \overline{a_{j}} \leq a_{i}-1$ etc. In other words, the proper fraction of $\sigma_{i}$ coincides with the image $R_{i}(\mathbf{a} / d)$ of the $i$-th remainder map.

PROOF. The multiplicity of $\sigma_{i}$ coincides with

$$
\left|\operatorname{det}\left(P_{1}, \ldots, P_{i-1}, \frac{\sum_{i=1}^{n-1} a_{i} P_{i}+P_{n}}{d}, P_{i+1}, \ldots, P_{n}\right)\right|=\frac{a_{i}}{d}\left|\operatorname{det}\left(P_{i}, \ldots, P_{n}\right)\right|=a_{i} .
$$

Since $Q_{i}$ is Oka center, there exist $x_{j}(j \neq i)$ and $x^{\prime}$ with $0 \leq x_{j} \leq a_{i}-1,0 \leq x^{\prime} \leq$ $a_{i}-1$ such that

$$
a_{i} Q_{i}=\sum_{j \neq i, n} x_{j} P_{j}+x^{\prime} Q+P_{n}
$$

It follows from (12) and (15) that

$$
a_{i} Q_{i}=\sum_{j \neq i, n}\left(x_{j}-a_{j}\right) P_{j}-a_{i} P_{i}+\left(x^{\prime}+d\right) Q .
$$


Since $Q_{i}$ is contained in the lattice $\mathbf{Z} P_{1}+\cdots+\mathbf{Z} P_{n-1}+\mathbf{Z} Q$, we have

$$
x_{j} \equiv a_{j}, x^{\prime} \equiv-d\left(\bmod a_{i}\right) .
$$

Therefore the assertion follows.

1.3. From now on, we assume that the proper fraction $\mathbf{a} / d$ of this semi-unimodular cone $\sigma$ is irreducible. We put $X:=U_{\sigma}=\mathrm{T}_{N} \mathrm{emb}\left(\Delta_{\sigma}\right)$. Then $X$ has an isolated orbipoint of type $\mathbf{a} / d$ at the origin. Moreover Oka center $Q$ of $\sigma$ is in the interior $\operatorname{supp}(\sigma)^{(0)}$ of $\sigma$, i.e., $\mathbf{R}_{\geq 0} Q$ is not contained in any proper face of $\sigma$.

Now let $\widetilde{\sigma}$ be the subdivision of $\sigma$ at the center $Q$. Then $\widetilde{\sigma}$ consists of $n$ simplicial cones. Let $\Delta \widetilde{\sigma}$ be the fan consisting of all the faces of $\widetilde{\sigma}$. We consider the natural birational equivariant morphism

$$
\mu: Y=\mathrm{T}_{N} \mathrm{emb}(\Delta \widetilde{\sigma}) \longrightarrow X .
$$

We call $\mu$ Fujiki-Oka modification of $X$. Note that this modification essentially appears in Fujiki [Fuj, Lemma 3] without using the terminology of toric geometry. Then $\mu$ has the following good compactification $\bar{\mu}: \bar{Y} \rightarrow \bar{X}$, the existence of whose proof is postponed to $\$ 3.3$

Proposition 1.3.1. Let $\overline{\Delta_{\sigma}}$ be a simplicial completion of $\Delta_{\sigma}$, and let $\overline{\Delta_{\tilde{\sigma}}}$ be the simplical completion of $\Delta_{\widetilde{\sigma}}$ which is obtained by the subdivision of $\sigma$ to $\widetilde{\sigma}$ in $\overline{\Delta_{\sigma}}$.

We can choose such a pair $\overline{\Delta_{\sigma}}, \overline{\Delta_{\tilde{\sigma}}}$ so that $\overline{\Delta_{\sigma}}$ and $\overline{\Delta_{\tilde{\sigma}}}$ are both projective. Namely $\bar{Y}=\mathrm{T}_{N} \mathrm{emb}\left(\overline{\Delta_{\tilde{\sigma}}}\right)$ and $\bar{X}=\mathrm{T}_{N} \mathrm{emb}\left(\overline{\Delta_{\sigma}}\right)$ are both projective.

2. Signature as rational homology manifold. For the compactified Fujiki-Oka $\bar{\mu}$ : $\bar{Y} \rightarrow \bar{X}$ as in $\S 1.3$, we describe the difference of the signatures Sign $\bar{Y}-\operatorname{Sign} \bar{X}$.

2.1. From now on to the last section ( $\$ \S 2-5)$, the natural number $n$ is assumed to be even otherwise stated.

Let $\Delta$ be an $n$-dimensional projective simplicial fan, and put $W=\mathrm{T}_{N} \mathrm{emb}(\Delta)$. Since $W$ is simplicial, $W$ is automatically a complex orbifold. Especially $W$ is a rational homology manifold (cf. [St, p. 527]). Let Sign $W$ be the signature of the intersection form on $H^{n}(W, \mathbf{Q})$ as a rational homology manifold.

For $0 \leq j \leq n$, let $\Delta(j)$ be the set of $j$-dimensional cones of $\Delta$ and put $|\Delta(j)|$ the cardinarity of $\Delta(j)$.

The following theorem is proved by Leung-Reiner [LR, Theorem 1.1] by using the result of McMullen [Mc, Theorem 8.6]. (See also [MS, §3] for its generalization.) Here we give another proof, whose method is analogous to [Od, §3.3].

THEOREM 2.1.1. ([LR], [Mc]). For a simplicial projective toric variety $W=$ $\mathrm{T}_{N} \mathrm{emb}(\Delta)$, we have

$$
\text { Sign } W=\sum_{j=0}^{n}(-2)^{j}|\Delta(n-j)| \text {. }
$$


Proof. Let $\operatorname{Sing}(W)$ be the singular locus of $W$ as an analytic space, and let $i: W-$ $\operatorname{Sing}(W) \hookrightarrow W$ be the inclusion map. Let $\Omega_{W-\operatorname{Sing}(W)}^{\bullet}$ be the de Rham complex on $W-$ $\operatorname{Sing}(W)$, and put $\widetilde{\Omega}_{W}^{\bullet}=i_{*} \Omega_{W-\operatorname{Sing}(W)}^{\bullet}$. By Danilov [D, Thm.12.5], the spectral sequence

$$
E_{1}^{p, q}=H^{q}\left(W, \widetilde{\Omega}_{W}^{p}\right) \Longrightarrow H^{p+q}(W, \mathbf{C})
$$

degenerates at $E_{1}$-term. Especially, by putting

$$
h^{p, q}(W)=\operatorname{dim} H^{q}\left(W, \widetilde{\Omega}_{W}^{p}\right),
$$

the $k$-th Betti number is written as $b_{k}(W)=\sum_{p+q=k} h^{p, q}(W)$.

By the projectivity of $W$, there exists an ample line bundle on $W$, and we denote by $L \in H^{2}(W, \mathbf{C})$ its cohomology class. By Beilinson-Bernstein-Deligne [BBD] and GoreskyMacPherson [GM] the hard Lefschetz theorem holds for $W$, i.e., the map $\omega \mapsto L^{q} \wedge \omega$ induces an isomorphism from $H^{n-q}(W, \mathbf{C})$ to $H^{n+q}(W, \mathbf{C})$ for any $q \in \mathbf{N}$ (see also [CLS, p. 620 and the comment in p. 794]). We denote the $k$-th primitive cohomology by

$$
P^{k}(W, \mathbf{C})=\operatorname{Ker}\left(L^{n-k+1}: H^{k}(W, \mathbf{C}) \rightarrow H^{2 n-k+1}(W, \mathbf{C})\right) .
$$

Then we have the Lefschetz decomposition

$$
H^{q}(W, \mathbf{C})=\oplus_{r \geq 0} L^{r} P^{q-2 r}(W, \mathbf{C})
$$

for $q \geq 0$. By Steenbrink [St, p. 531], the Hermitian inner product on $H^{n-q}(W, \mathbf{C})$ defined by

$$
Q(x, y)=(-1)^{(n-q)(n-q-1) / 2} C x \wedge L^{q} \bar{y}[W]
$$

is positive definite, where $C$ is the Weil operator and $\bar{y}$ is the complex conjugation of $y$ with respect to $H^{n-q}(W, \mathbf{R})$. Therefore by the same argument as in [GH, pp. 125-126], we have the "Hirzebruch-type" formula

$$
\text { Sign } W=\sum_{p+q \equiv 0 \bmod 2}(-1)^{q} h^{p, q}(W) .
$$

On the other hand, we define the Poincaré series of $W$ as

$$
P(t)=\sum_{p=0}^{n} h^{p, p}(W) t^{p} \in \mathbf{Z}[t] .
$$

By using the Ishida complex [I1] for a simplicial toric variety, Oda [Od, Thm.3.11,(2)] shows that

$$
P(t)=\sum_{j=0}^{n}|\Delta(j)|(t-1)^{n-j} .
$$

Since $h^{p, q}(W)=0$ for $p \neq q$ (cf. [D, Cor.12.7], [Od, Thm.3.11, (1)]), it follows from (16) that Sign $W$ coincides with $P(-1)$. Therefore the assersion follows from (17) by putting $t=-1$. 
COROLlary 2.1.2. The compactified Fujiki-Oka modification $\bar{\mu}: \bar{Y} \longrightarrow \bar{X}$ satisfies

$$
\text { Sign } \bar{Y}-\operatorname{Sign} \bar{X}=-1 \text {. }
$$

Proof. Since $\bar{X}$ and $\bar{Y}$ have the same fans outsides $\sigma$ and the subdivision $\widetilde{\sigma}$ of $\sigma$ is barycentric, it follows from Theorem 2.1.1 that

$$
\begin{aligned}
\operatorname{Sign} \bar{Y}-\operatorname{Sign} \bar{X} & =\sum_{j=0}^{n-1}(-2)^{j}\left(|\Delta \widetilde{\sigma}(n-j)|-\left|\Delta_{\sigma}(n-j)\right|\right) \\
& =\sum_{j=0}^{n-1}(-2)^{j}{ }_{n} C_{j+1}=\frac{-(1-2)^{n}-1}{2}=-1 .
\end{aligned}
$$

3. Orbifold signature. In this section, we describe the difference of the orbifold signatures $\operatorname{Sign}^{\text {orb }} \bar{Y}-\operatorname{Sign}^{\text {orb }} \bar{X}$ for the modification $\bar{\mu}: \bar{Y} \rightarrow \bar{X}$. For this purpose, we calculate the difference between rational Chow rings $A^{*}(\bar{Y})_{\mathbf{Q}}$ and $A^{*}(\bar{X})_{\mathbf{Q}}$. By using this, we also prove the claim of the projectivity in Proposition 1.3.1.

3.1. For an $n$-dimensional simplicial toric variety $W=\mathrm{T}_{N} \mathrm{emb}(\Delta)$, let $A^{*}(W)_{\mathbf{Q}}$ be the rational Chow ring of $W$. For each $\rho \in \Delta$, let $V(\rho)$ be the $T_{N}$-invariant subvariety corresponding to $\rho$, i.e., $V(\rho)$ is the closure $\overline{\operatorname{orb}(\rho)}$ of the $T_{N}$-orbit corresponding to $\rho$. By essentially Jurkewicz and Danilov [D, p.131], $A^{*}(W)_{\mathbf{Q}}$ is generated by the $T_{N}$-invariant divisors $\{V(\rho) ; \rho \in \Delta(1)\}$ with the following relations:

(i) For any $m \in W$, we have

$$
\sum_{\rho \in \Delta(1)}\langle m, \rho\rangle V(\rho)=0 .
$$

(ii) If $\rho_{1}, \ldots, \rho_{k} \in \Delta(1)$ are mutually distinct, then

$$
\begin{aligned}
& V\left(\rho_{1}\right) \cdot V\left(\rho_{2}\right) \cdots V\left(\rho_{k}\right) \\
& \quad= \begin{cases}\frac{1}{\operatorname{mult}\left(\rho_{1}+\cdots+\rho_{k}\right)} V\left(\rho_{1}+\cdots+\rho_{k}\right) & \text { if } \rho_{1}+\cdots+\rho_{k} \in \Delta \\
0 & \text { otherwise, }\end{cases}
\end{aligned}
$$

where $\rho_{1}+\cdots+\rho_{k}$ is the face of $\Delta$ generated by $\rho_{1}, \ldots, \rho_{k}$.

The rational total Chern class of $W$ is given by

$$
c_{\mathbf{Q}}(W)=\prod_{\rho \in \Delta(1)}(1+V(\rho)) \in A^{*}(W)_{\mathbf{Q}}=\sum_{\rho \in \Delta} \frac{1}{\operatorname{mult}(\rho)} V(\rho),
$$

and the orbifold signature of $W$ is the rational number defined by

$$
\operatorname{Sign}^{\text {orb }}(W)=\kappa_{n}\left(\prod_{\rho \in \Delta(1)} V(\rho) t \cdot \operatorname{coth}(V(\rho) t)\right) \in A^{n}(W)_{\mathbf{Q}} \simeq \mathbf{Q} .
$$


As will be discussed in $\$ 5.1$, the definition (20) coincides with the V-signature of Satake [Sa].

3.2. We go back to the compactified Fujiki-Oka modification $\bar{\mu}: \bar{Y} \longrightarrow \bar{X}$. The following argument is analogous to that of Pommersheim [P, §3].

PROPOSITION 3.2.1.

$$
\operatorname{Sign}^{\text {orb }}(\bar{Y})-\operatorname{Sign}^{\text {orb }}(\bar{X})=-\frac{L_{n}\left(d, a_{1}, \ldots, a_{n-1}, 1\right)}{d \prod_{i=1}^{n-1} a_{i}} .
$$

Proof. As in the proof of Proposition 1.2.3, let $\left\{m_{1}, \ldots, m_{n}\right\}$ be the $\mathbf{Z}$-basis dual to the $\mathbf{Z}$-basis $\left\{P_{1}, \ldots, P_{n-1}, Q\right\}$ of the lattice $N$ in this order. Let $\left\{P_{1}, \ldots, P_{n}, P_{n+1}, \ldots, P_{s}\right\}$ be the primitive elements of the rays of $\overline{\Delta_{\sigma}}$ so that $\left\{P_{n+1}, \ldots, P_{s}\right\}$ are lying on $\mathbf{R}^{n} \backslash \operatorname{supp}(\sigma)$. Then $\left\{Q, P_{1}, \ldots, P_{n}, P_{n+1}, \ldots, P_{s}\right\}$ are the primitive elements of rays of $\overline{\Delta_{\tilde{\sigma}}}$, and the rational total Chern classes are

$$
c_{\mathbf{Q}}(\bar{X})=\prod_{i=1}^{s}\left(1+V\left(P_{i}\right)_{\bar{X}}\right), \quad c_{\mathbf{Q}}(\bar{Y})=(1+V(Q)) \prod_{i=1}^{s}\left(1+V\left(P_{i}\right)_{\bar{Y}}\right),
$$

where $V\left(P_{i}\right)_{\bar{X}}$ (resp. $\left.V\left(P_{i}\right)_{\bar{Y}}\right)$ are the rational equivalence class of $V\left(\mathbf{R}_{\geq 0} P_{i}\right)$ in $A^{*}(\bar{X})_{\mathbf{Q}}$ (resp. $A^{*}(\bar{Y})_{\mathbf{Q}}$ ), etc. Now it follows from (12) and (18) that

$$
\begin{aligned}
& 0=\left\langle m_{n}, Q\right\rangle V(Q)+\sum_{i=1}^{s}\left\langle m_{n}, P_{i}\right\rangle V\left(P_{i}\right)_{\bar{Y}}=V(Q)+d V\left(P_{n}\right)_{\bar{Y}}+\varepsilon_{n}, \\
& 0=\left\langle m_{j}, Q\right\rangle V(Q)+\sum_{i=1}^{s}\left\langle m_{j}, P_{i}\right\rangle V\left(P_{i}\right)_{\bar{Y}}=V\left(P_{j}\right)-a_{i} V\left(P_{i}\right)_{\bar{Y}}+\varepsilon_{j} \quad(1 \leq j \leq n-1),
\end{aligned}
$$

where $\varepsilon_{j}=\sum_{i=n+1}^{s}\left\langle m_{j}, P_{i}\right\rangle V\left(P_{i}\right)_{\bar{Y}}$ for $1 \leq j \leq n$. Therefore on $A^{*}(\bar{Y})_{\mathbf{Q}}$, we have

$$
V\left(P_{n}\right)_{\bar{Y}}=-\frac{1}{d} \cdot V(Q)-\frac{\varepsilon_{n}}{d}, V\left(P_{j}\right)_{\bar{Y}}=-\frac{a_{j}}{d} \cdot V(Q)-\frac{a_{j} \varepsilon_{n}}{d}+\varepsilon_{j} \quad(1 \leq j \leq n-1) .
$$

On $A^{*}(\bar{X}) \mathbf{Q}$, we similarly have

$$
V\left(P_{n}\right)_{\bar{X}}=-\frac{\varepsilon_{n}}{d}, \quad V\left(P_{j}\right)_{\bar{Y}}=-\frac{a_{j} \varepsilon_{n}}{d}+\varepsilon_{j} \quad(1 \leq j \leq n-1) .
$$

Here $\varepsilon_{j}=\sum_{i=n+1}^{s}\left\langle m_{j}, P_{i}\right\rangle V\left(P_{i}\right)_{\bar{X}}$ in (23) more precisely, although we use the same symbol $\varepsilon_{j}$ as in (22) by identifying them through the isomorphism $\bar{Y} \backslash \operatorname{supp}(\widetilde{\sigma}) \longrightarrow \bar{X} \backslash \operatorname{supp}(\sigma)$.

For $n+1 \leq i \leq s$, since $P_{i}$ and $Q$ are not in a same simplicial cone in $\overline{\Delta_{\tilde{\sigma}}}$, we have $V(Q) V\left(P_{i}\right)=0$. Especially

$$
V(Q) \cdot \varepsilon_{j}=0 \quad(1 \leq j \leq n)
$$


By an easy calculation using (21)-(24), we have

$$
\begin{aligned}
\operatorname{Sign}^{\text {orb }}(\bar{Y})-\operatorname{Sign}^{\text {orb }}(\bar{X}) \\
=\kappa_{n}\left\{V(Q) t \cdot \operatorname{coth}(V(Q) t) \cdot \prod_{j=1}^{n-1}\left(\left(-\frac{a_{j}}{d} V(Q) t\right) \operatorname{coth}\left(-\frac{a_{j}}{d} V(Q) t\right)\right)\right. \\
\left.\quad \times\left(-\frac{1}{d} V(Q) t\right) \operatorname{coth}\left(-\frac{1}{d} V(Q) t\right)\right\} \\
=V(Q)^{n} \cdot L_{n}\left(1,-\frac{a_{1}}{d}, \ldots,-\frac{a_{n-1}}{d},-\frac{1}{d}\right) \\
=\frac{V(Q)^{n}}{d^{n}} \cdot L_{n}\left(d, a_{1}, \ldots, a_{n-1}, 1\right) .
\end{aligned}
$$

Since the simplicial cone $\mathbf{R}_{\geq 0} P_{1}+\cdots+\mathbf{R}_{\geq 0} P_{n-1}+\mathbf{R}_{\geq 0} Q$ is nonsingular, it follows from (19), (22), (24) that

$$
1=V(Q) V\left(P_{1}\right) \cdots V\left(P_{n-1}\right)=V(Q)^{n} \cdot \frac{(-1)^{n-1} a_{1} \cdots a_{n-1}}{d^{n-1}} .
$$

Namely

$$
V(Q)^{n}=-\frac{d^{n-1}}{a_{1} \cdots a_{n-1}} .
$$

From (25), (26), the assertion follows.

REMARK 3.2.2. From the argument in the proof of Proposition 3.2.1, we have the following: Let $\mathbf{Q}\left[\mathbf{q}, \mathbf{p}_{1}, \ldots, \mathbf{p}_{n}\right]$ be the polynomial ring in $n+1$ variables $\mathbf{q}, \mathbf{p}_{1}, \ldots, \mathbf{p}_{n}$ with rational coefficients, and let $f\left(\mathbf{q}, \mathbf{p}_{1}, \ldots, \mathbf{p}_{n}\right) \in \mathbf{Q}\left[\mathbf{q}, \mathbf{p}_{1}, \ldots, \mathbf{p}_{n}\right]$ be the homogeneous term of degree $n$ of the power series of the function $\mathbf{q} \operatorname{coth} \mathbf{q} \cdot\left(\prod_{i=1}^{n} \mathbf{p}_{i} \operatorname{coth} \mathbf{p}_{i}\right)$. Then the invariant $\operatorname{Sign}^{\text {orb }}(\bar{Y})-\operatorname{Sign}^{\text {orb }}(\bar{X})$ is nothing but the rational number obtained from $f\left(\mathbf{q}, \mathbf{p}_{1}, \ldots, \mathbf{p}_{n-1}, \mathbf{p}_{n}\right)$ by substituting the relation

$$
\mathbf{p}_{i}=-\frac{a_{i}}{d} \mathbf{q} \quad(1 \leq i \leq n-1), \quad \mathbf{p}_{n}=-\frac{1}{d} \mathbf{q}, \quad \mathbf{q}^{n}=-\frac{d^{n-1}}{a_{1} \cdots a_{n-1}} .
$$

3.3. We prove Proposition 1.3.1. The existence of simplicial completions of $X, Y$ is obvious. Since the discussion in $\$ 3.2$ is independent of the projectivity of $\bar{X}, \bar{Y}$, we can use the results in $\$ 3.2$.

Let $\ell_{\sigma}$ be a $|\sigma|$-linear support function of $\sigma$, and let $\left[\ell_{\sigma}\right]$ be the element of the dual lattice $M$ which corresponds to $\ell_{\sigma}$. Let $\mathcal{P}$ be an integral polytope containing $\left[\ell_{\sigma}\right]$ as one of the vertices. By the Galois correspondence [Od, §2.4], there exists a projective fan $\bar{\Delta}_{\sigma}$ which corresponds to $\mathcal{P}$, i.e., $\bar{X}=\mathrm{T}_{N} \mathrm{emb}\left(\bar{\Delta}_{\sigma}\right)$ is projective.

Now let $\bar{\Delta}_{\widetilde{\sigma}}$ be the fan which is obtained from the Oka subdivision of the simplicial cone $\sigma$ in $\bar{\Delta}_{\sigma}$. It suffices to prove that $\bar{\Delta}_{\widetilde{\sigma}}$ is also a projective fan.

For a $\mathbf{Q}$-ample divisor $D_{1}$ and an effective divisor $D_{2}$ on a complex orbifold in general, the divisor $r_{1} D_{1}+r_{2} D_{2} \quad\left(r_{1}, r_{2} \in \mathbf{Q}_{\geq 0}\right)$ is also $\mathbf{Q}$-ample. Therefore there exists a set of 
positive rational numbers $\left\{\alpha_{i}\right\}_{1 \leq i \leq s}$ such that for any set of rational numbers $\left\{x_{i}\right\}_{1 \leq i \leq s}$ which satisfy

$$
x_{i} \geq \alpha_{i} \quad(1 \leq i \leq s),
$$

the divisor $\sum_{i=1}^{s} x_{i} V\left(P_{i}\right)_{\bar{X}}$ is $\mathbf{Q}$-ample on $\bar{X}$.

Next we deal with $\bar{Y}$. We set $P_{0}=Q$ and $V\left(P_{i}\right)=V\left(P_{i}\right)_{\bar{Y}}$ for simplicity. We claim that, if we choose some rational numbers $\left\{x_{i}\right\}_{1 \leq i \leq s}$ which satisfiy (28), then there exists a rational number $x_{0} \in \mathbf{Q}_{\geq 0}$ such that the divisor

$$
D=\sum_{i=0}^{s} x_{i} V\left(P_{i}\right)
$$

is $\mathbf{Q}$-ample on $\bar{Y}$.

Let $C$ be any $\mathrm{T}_{\mathrm{N}}$-invariant curve on $\bar{Y}$. In order to prove the ampleness of $D$, it suffices to prove $D C>0$ by the toric Nakai criterion [Od, p. 95]. The curve $C$ is written as $V\left(P_{j_{1}}\right) \cdots V\left(P_{j_{n-1}}\right)$ for some $0 \leq j_{1}<\cdots<j_{n-1} \leq s$. Our discussion is divided into the following cases.

(i) Assume $j_{n-1} \geq n+1$. Then $C$ is contained in the closure of $\bar{Y} \backslash \operatorname{supp} \widetilde{\sigma}$, and therefore $C \rightarrow \bar{\mu}(C)$ is an isomorphism. Since we may assume that $\bar{\mu}_{*}(D)=\sum_{i=1}^{s} x_{i} V\left(P_{i}\right)_{\bar{X}}$ is ample on $\bar{X}$ by (28), we have $D C=\bar{\mu}_{*}(D) \bar{\mu}_{*}(C)>0$.

(ii) Assume $j_{n-1} \leq n$. Our discussion is divided into the following sub-cases.

(ii-a) Suppose $j_{1}=0$. Then $C$ is written as $V\left(P_{0}\right) \cdots V\left(P_{i-1}\right) V\left(P_{i+1}\right) \cdots V\left(P_{j-1}\right)$ $V\left(P_{j+1}\right) \cdots V\left(P_{n}\right)$ for some $1 \leq i<j \leq n$. Since $V\left(P_{0}\right) V\left(P_{k}\right)=0$ in $A^{*}(\bar{Y})_{\mathbf{Q}}$ for $k \geq n+1$, we have $D C=\sum_{k=0}^{n} V\left(P_{k}\right) C$. Moreover in this case, the equations (22), (24), (26) say that we may numerically assume

$$
V\left(P_{k}\right) \equiv-\frac{a_{k}}{d} \cdot V\left(P_{0}\right) \quad(1 \leq k \leq n), \quad V\left(P_{0}\right)^{n} \equiv-\frac{d^{n-1}}{a_{1} \cdots a_{n}} .
$$

By an easy calculation using (30), we have

$$
D C=\frac{1}{a_{i} a_{j}}\left(\sum_{k=1}^{n} a_{k} x_{k}-d x_{0}\right) .
$$

Hence the condition $D C>0$ is equivalent to

$$
\sum_{k=1}^{n} a_{k} x_{k}-d x_{0}>0
$$

(ii-b) Suppose $j_{1} \geq 1$. Then $C$ is written as $V\left(P_{1}\right) \cdots V\left(P_{i-1}\right) V\left(P_{i+1}\right) \cdots V\left(P_{n}\right)$ for some $1 \leq i \leq n$. By an easy calculation using (22), (24), (26), we have

$$
\begin{aligned}
D C= & \frac{1}{a_{i} d}\left(-\sum_{k=1}^{n} a_{k} x_{k}+d x_{0}\right)+\sum_{k=1}^{n} x_{k} D_{1} \cdots D_{n} \\
& +\sum_{k=n+1}^{s} x_{k} V\left(P_{k}\right) V\left(P_{1}\right) \cdots V\left(P_{i-1}\right) V\left(P_{i+1}\right) \cdots V\left(P_{n}\right),
\end{aligned}
$$


where $D_{1}, \ldots, D_{n}$ are some $\mathbf{Q}$-divisors whose supports are contained in the support of $\sum_{k=n+1}^{s} V\left(P_{k}\right)$. Therefore the condition $D C>0$ is equivalent to

$$
\begin{gathered}
\sum_{k=n+1}^{s} x_{k} V\left(P_{k}\right) V\left(P_{1}\right) \cdots V\left(P_{i-1}\right) V\left(P_{i+1}\right) \cdots V\left(P_{n}\right) \\
>\frac{1}{a_{i} d}\left(\sum_{k=1}^{n} a_{k} x_{k}-d x_{0}\right)-\sum_{k=1}^{n} x_{k} D_{1} \cdots D_{n} .
\end{gathered}
$$

The linear inequalties (28), (31), (32) with respect to $x_{0}, x_{1}, \ldots, x_{s}$ have simultaneous rational solutions. Indeed, as the first step, we choose $x_{0}, x_{1}, \ldots, x_{n}$ so that (28) (31) are satisfied. For any $i \quad(1 \leq i \leq n)$, there exists some $k(n+1 \leq k \leq s)$ such that $\mathbf{R}_{\geq 0} P_{k}+$ $\mathbf{R}_{\geq 0} P_{1}+\cdots+\mathbf{R}_{\geq 0} P_{i-1}+\mathbf{R}_{\geq 0} P_{i+1}+\cdots+\mathbf{R}_{\geq 0} P_{n}$ is a simplicial cone in $\bar{\Delta} \tilde{\sigma}$. Therefore, as the second step, we can choose $x_{n+1}, \ldots, x_{s}$ sufficiently large so that (32) is also satisfied.

4. Reciprocity of Dedekind sums with weight. By using Atiyah-Singer cotangent function, we define the notion of Dedekin sums with weight and state our reciprocity.

4.1. We set $\mathrm{I}=\{1,2, \ldots, n\}$. For $\mathbf{a} / d \in \mathbf{Q}_{n}^{\text {prop }}$ as in (6), we denote by I the disjoint union $\mathrm{I}_{0}(\mathbf{a} / d) \bigsqcup \mathrm{I}_{\text {ref }}(\mathbf{a} / d) \bigsqcup \mathrm{I}_{*}(\mathbf{a} / d)$, where

$$
\begin{aligned}
& \mathrm{I}_{0}(\mathbf{a} / d)=\left\{i \in \mathrm{I} ; a_{i}=0\right\}, \quad \mathrm{I}_{\mathrm{ref}}(\mathbf{a} / d)=\left\{i \in \mathrm{I} ; \frac{a_{i}}{d}=\frac{1}{2}\right\}, \\
& \mathrm{I}_{*}(\mathbf{a} / d)=\mathrm{I} \backslash\left(\mathrm{I}_{0}(\mathbf{a} / d) \coprod \mathrm{I}_{\mathrm{ref}}(\mathbf{a} / d)\right) .
\end{aligned}
$$

For a fixed $\mathbf{a} / d$, we sometimes simply write $\mathrm{I}_{0}=\mathrm{I}_{0}(\mathbf{a} / d)$ and so on if there is no confusion. We denote by $\left|\mathrm{I}_{0}\right|$ the cardinality of $\mathrm{I}_{0}$ and so on.

For a Laurent series $f\left(x_{1}, \ldots, x_{n}\right)=\sum_{-\infty<i_{1}, \ldots, i_{n}<\infty} a_{i_{1} \cdots i_{n}} x_{1}^{i_{1}} \cdots x_{n}^{i_{n}}$ in the variables $x_{1}, \ldots, x_{n}$ with rational coefficients, we denote by

$$
\kappa_{m}\left(f\left(x_{1}, \ldots, x_{n}\right)\right)=\sum_{i_{1}+\cdots+i_{n}=m} a_{i_{1} \cdots i_{n}} x_{1}^{i_{1}} \cdots x_{n}^{i_{n}} \quad(m \in \mathbf{Z})
$$

its homogeneous part of degree $m$. For simplicity, we write $\mathbf{x}=\left(x_{1}, \ldots, x_{n}\right)$.

Definition 4.1.1. For $\mathbf{a} / d \in \mathbf{Q}_{n}^{\text {prop }}$, Atiyah-Singer cotangent function $\cot (\mathbf{a} / d)(\mathbf{x})$ is the meromorphic function defined by

$$
\frac{(-1)^{n} \cdot 2^{\left|\mathrm{I}_{0}\right|-\left|\mathrm{I}_{\mathrm{ref}}\right|}}{d\left(\left|\mathrm{I}_{0}\right|+1\right)\left(\prod_{k \in \mathrm{I}_{0}} x_{k}\right)} \cdot \kappa_{\left|\mathrm{I}_{0}\right|}\left\{\prod_{k \in \mathrm{I}_{*}} \operatorname{coth}\left(\frac{x_{k}}{2}+\frac{\sqrt{-1} \pi a_{k}}{d}\right) \prod_{k \in \mathrm{I}_{0}} \frac{x_{k}}{2} \operatorname{coth} \frac{x_{k}}{2} \prod_{k \in \mathrm{I}_{\mathrm{ref}}} 2 \tanh \frac{x_{k}}{2}\right\} \text {. }
$$

This function appears as an interpretation in our situation of Atiyah-Singer's equivariant L-class [AS, p. 582]. Taylor expansion says

$$
(-1)^{n} 2^{\left|\mathrm{I}_{\mathrm{ref}}\right|-\left|\mathrm{I}_{0}\right|} d\left(\left|\mathrm{I}_{0}\right|+1\right)\left(\prod_{k \in \mathrm{I}_{0}} x_{k}\right) \cot (\mathbf{a} / d)(\mathbf{x})
$$


is its homogeneous part of degree $\left|\mathrm{I}_{0}\right|$ of

$$
\begin{aligned}
& \prod_{k \in \mathrm{I}_{*}} \sum_{j=0}^{\infty} \frac{(-\sqrt{-1})^{j+1}}{j ! 2^{j}} \cot ^{(j)}\left(\frac{\pi a_{k}}{d}\right) x_{k}^{j} \\
& \cdot \prod_{k \in \mathrm{I}_{0}} \sum_{j=0}^{\infty} \frac{B_{2 j}}{(2 j) !} x_{k}^{2 j} \cdot \prod_{k \in \mathrm{I}_{\mathrm{ref}}} \sum_{j=1}^{\infty} \frac{4\left(2^{2 j}-1\right) B_{2 j}}{(2 j) !} x_{k}^{2 j-1},
\end{aligned}
$$

where $B_{2 j}$ is $2 \mathrm{j}$-th Bernoulli number so that $B_{0}=1, B_{2}=1 / 6, B_{4}=-1 / 30, \ldots$, and $\cot ^{(j)} x=\left(d^{j} / d x^{j}\right)(\cot x)$ (see e.g. [B] for the notation). Therefore Atiyah-Singer cotangent function is essentially written in terms of the derivatives of the usual cotangent function and Bernoulli numbers.

EXAMPLE 4.1.2. If $\mathrm{I}_{0}(\mathbf{a} / d)=\emptyset$, then $\cot (\mathbf{a} / d)(\mathbf{x})$ is the constant function

$$
\cot \left(\frac{\mathbf{a}}{d}\right)(\mathbf{x})=\frac{(-1)^{n / 2}}{d} \prod_{k=1}^{n} \cot \left(\frac{\pi a_{k}}{d}\right) .
$$

EXAMPLE 4.1.3. If $\mathbf{a} / d=\left(a_{1}, a_{2}, 0,0\right) / d \in \mathbf{Q}_{4}^{\text {prop }}$ with $d \geq 3, \operatorname{gcd}\left(d, a_{1}\right)=$ $\operatorname{gcd}\left(d, a_{2}\right)=1$, then

$$
\begin{aligned}
\cot \left(\frac{\mathbf{a}}{d}\right)(\mathbf{x})= & \frac{1}{18 d x_{3} x_{4}}\left(3 x_{1}^{2} \cot ^{(2)} \frac{\pi a_{1}}{d} \cot \frac{\pi a_{2}}{d}+6 x_{1} x_{2} \cot ^{(1)} \frac{\pi a_{1}}{d} \cot ^{(1)} \frac{\pi a_{2}}{d}\right. \\
& \left.+3 x_{2}^{2} \cot \frac{\pi a_{1}}{d} \cot ^{(2)} \frac{\pi a_{2}}{d}-2\left(x_{3}^{2}+x_{4}^{2}\right) \cot \frac{\pi a_{1}}{d} \cot \frac{\pi a_{2}}{d}\right) .
\end{aligned}
$$

4.2. We define the following.

Definition 4.2.1. For $\mathbf{a} / d \in \mathbf{Q}_{n}^{\text {prop }}$ and an element $\mathbf{w}=\left(w_{1}, \ldots, w_{n}\right) \in \mathbf{Z}^{n}$, we define the Dedekind sum of $\mathbf{a} / d$ with weight $\mathbf{w}$ by

$$
\mathbf{s}\left(\frac{\mathbf{a}}{d}, \mathbf{w}\right)=\sum_{k=1}^{d-1} \cot \left(k \cdot \frac{\mathbf{a}}{d}\right)(\mathbf{w}) .
$$

If $\mathbf{a} / d$ is irreducible, then $k \cdot \mathbf{a} / d$ is also irreducible for $1 \leq k \leq d-1$. In this case, Example 4.1.2 says that

$$
\mathbf{s}\left(\frac{\mathbf{a}}{d}, \mathbf{w}\right)=\frac{(-1)^{n / 2}}{d} \cdot \sum_{k=1}^{d-1} \prod_{i=1}^{n} \cot \frac{\pi a_{i} k}{d}
$$

independently of the weight $\mathbf{w}$, and we simply write it as $\mathbf{s}(\mathbf{a} / d)$. The definition (36) for an irreducible fraction $\mathbf{a} / d$ is essentially nothing but the Dedekind sum defined by HirzebruchZagier [HZ]. In this sense, the notion of Dedekind sums with weight is a generalization of the one in [HZ]. Now let

$$
L_{n}\left(x_{1}, \ldots, x_{r}\right)=\kappa_{n}\left(\prod_{i=1}^{r} x_{i} \operatorname{coth} x_{i}\right)
$$


be the Hirzebruch L-polynomial in the variables $x_{1}, \ldots, x_{r}$. The following theorem is our main result, whose proof will be accomplished in $\$ 5$.

THEOREM 4.2 .2 (Reciprocity law). Let $\mathbf{a} / d=\left(a_{1}, \ldots, a_{n-1}, 1\right) / d \in \mathbf{Q}_{n}^{\text {prop }} \quad$ be an irreducible inhomogeneous element. Then we have

$$
\mathbf{s}\left(\frac{\mathbf{a}}{d}\right)-\sum_{i=1}^{n-1} \mathbf{s}\left(R_{i}\left(\frac{\mathbf{a}}{d}\right), N_{i}\left(\frac{\mathbf{a}}{d}\right)\right)=1-\frac{L_{n}\left(d, a_{1}, \ldots, a_{n-1}, 1\right)}{d \prod_{i=1}^{n-1} a_{i}} .
$$

If $\mathbf{a} / d$ is absolutely irreducible, then $R_{i}(\mathbf{a} / d)$ is also (absolutely) irreducible for any $i$. Therefore Theorem 4.2.2 implies the following:

Corollary 4.2.3. If $\mathbf{a} / d=\left(a_{1}, \ldots, a_{n-1}, 1\right) / d \in \mathbf{Q}_{n}^{\text {prop }} \quad$ is an absolutely irreducible inhomogeneous element, then

$$
\mathbf{s}\left(\frac{\mathbf{a}}{d}\right)-\sum_{i=1}^{n-1} \mathbf{s}\left(R_{i}\left(\frac{\mathbf{a}}{d}\right)\right)=1-\frac{L_{n}\left(d, a_{1}, \ldots, a_{n-1}, 1\right)}{d \prod_{i=1}^{n-1} a_{i}} .
$$

We easily check that Corollary 4.2.3 coincides with the inhomogeneous Zagier reciprocity. Namely if we put $a_{n}=1$ in the formula (47) in [Z, p. 158], then it coincides with (38). In this sense, the formula (37) is a generalization of the inhomogeneous Zagier reciprocity.

EXAMPLE 4.2.4. If $\mathbf{a} / d=\left(a_{1}, a_{2}, a_{3}, a_{4}\right) / d \in \mathbf{Q}_{4}^{\text {prop }}$, where $a_{4}=1, \operatorname{gcd}\left(d, a_{i}\right)=1$ $(1 \leq i \leq 3)$ and $d \geq 3$ is odd, then

$$
\mathbf{s}\left(\frac{\mathbf{a}}{d}\right)-\sum_{i=1}^{3} \mathbf{s}\left(R_{i}\left(\frac{\mathbf{a}}{d}\right), N_{i}\left(\frac{\mathbf{a}}{d}\right)\right)=1-\frac{1}{45 d \prod_{i=1}^{4} a_{i}}\left(\sum_{1 \leq i<j \leq 4} 15 a_{i}^{2} a_{j}^{2}-\sum_{i=1}^{4} a_{i}^{4}\right) .
$$

Here by putting $c_{1 i}=\operatorname{gcd}\left(a_{1}, a_{i}\right)(i=2,3), c_{123}=\operatorname{gcd}\left(a_{1}, a_{2}, a_{3}\right)$ and $d^{\prime}=-d$, we have

$$
\begin{aligned}
& \mathbf{s}\left(R_{1}\left(\frac{\mathbf{a}}{d}\right), N_{1}\left(\frac{\mathbf{a}}{d}\right)\right)=\frac{1}{a_{1}} \sum_{1 \leq k \leq a_{1}-1}^{\prime} \cot \frac{\pi d^{\prime} k}{a_{1}} \cot \frac{\pi a_{2} k}{a_{1}} \cot \frac{\pi a_{3} k}{a_{1}} \cot \frac{\pi k}{a_{1}} \\
& \quad+\sum_{i=2}^{3} \frac{1}{2 a_{1} a_{i}} \sum_{1 \leq k \leq c_{1 i}-1}^{\prime}\left(d^{\prime} \cot ^{(1)} \frac{\pi d^{\prime} k}{c_{1 i}} \cot \frac{\pi a_{i^{*} k}}{c_{1 i}} \cot \frac{\pi k}{c_{1 i}}\right. \\
& +a_{i^{*}} \cot \frac{\pi d^{\prime} k}{c_{1 i}} \cot ^{(1)} \frac{\pi a_{i^{*} k}}{c_{1 i}} \cot \frac{\pi k}{c_{1 i}} \\
& \left.+\cot \frac{\pi d^{\prime} k}{c_{1 i}} \cot \frac{\pi a_{i^{*} k}}{c_{1 i}} \cot ^{(1)} \frac{\pi k}{c_{1 i}}\right)+\frac{1}{18 a_{1} a_{2} a_{3}} \sum_{k=1}^{c_{123}-1}\left\{3 d^{\prime 2} \cot ^{(2)} \frac{\pi d^{\prime} k}{c_{123}} \cot \frac{\pi k}{c_{123}}\right. \\
& \left.+6 d^{\prime} \cot ^{(1)} \frac{\pi d^{\prime} k}{c_{123}} \cot ^{(1)} \frac{\pi k}{c_{123}}+3 \cot \frac{\pi d^{\prime} k}{c_{123}} \cot ^{(2)} \frac{\pi k}{c_{123}}-2\left(a_{2}^{2}+a_{3}^{2}\right) \cot \frac{\pi d^{\prime} k}{c_{123}} \cot \frac{\pi k}{c_{123}}\right\} .
\end{aligned}
$$


In the first (dashed) summation $\sum^{\prime}, k$ moves from 1 to $a_{1}-1$ such that $(\alpha k) / a_{1} \notin \mathbf{Z}$ for $\alpha=a_{2}, a_{3}$. In the second (dashed) summation $\sum^{\prime}$, the suffix $i^{*}$ is determined by $2^{*}=3$ and $3^{*}=2$, and $k$ moves from 1 to $c_{1 i}-1$ such that $\left(a_{i *} k\right) / c_{1 i} \notin \mathbf{Z}$.

The terms $\mathbf{s}\left(R_{i}(\mathbf{a} / d), N_{i}(\mathbf{a} / d)\right)$ for $i=2,3$ are similar.

5. S-defect. In this section, we calculate the difference $\operatorname{Sdef}(\bar{Y})-\operatorname{Sdef}(\bar{X})$ of the singature defects for the modification $\bar{\mu}: \bar{Y} \rightarrow \bar{X}$, and prove Theorem 4.2.2.

5.1. Let $Z$ be an $n$-dimensional complex abelian orbifold. Namely there exists an orbifold chart

$$
Z=\bigcup U_{i}, \quad\left\{\widetilde{U}_{i} \stackrel{\pi_{i}}{\longrightarrow} U_{i}, G_{i}=\operatorname{Gal}\left(\widetilde{U_{\sigma_{i}}} / U_{i}\right)\right\}
$$

so that any $G_{i}$ is an abelian group. First, we briefly recall the $\mathrm{V}$-signature theorem of Kawasaki [Ka] restricted to $Z$ for later use.

Let $L\left(\widetilde{U}_{i}\right)$ be the Hirzebruch L-form on $\widetilde{U}_{i}$. Let $\left\{\widetilde{\rho}_{i}, \rho_{i}\right\}$ be the partition of unity subordinate to the chart (39). Namely, $\widetilde{\rho}_{i}$ is a $C^{\infty}$ function whose support is contained in $\widetilde{U}_{i}$ so that the system of function $\rho_{i}$ on $U_{i}$ with $\pi_{i}^{*} \rho_{i}=\tilde{\rho}_{i}$ satisfies $\sum \rho_{i}=1$. Satake's V-signature ([Sa], [Ka]) of $Z$ is defiend by

$$
\operatorname{Sign}^{V}(Z)=\sum \frac{1}{\left|G_{i}\right|} \int_{\widetilde{U}_{i}} \tilde{\rho}_{i} L\left(\widetilde{U}_{i}\right) .
$$

The right-hand side of (40) is sometimes written as $\int_{Z}^{\text {orb }} L(Z)$.

On the other hand, for any $g \in G_{i}$, let $L\left(g, \widetilde{U}_{i}\right)$ be the equivariant L-form on $\widetilde{U}_{i}$ with respect to $g$ (cf. [AS]). The S-defect of $Z$ is defined by

$$
\operatorname{Sdef}(Z)=\sum_{i} \frac{1}{\left|G_{i}\right|} \sum_{g \in G_{i}, g \neq i d} \int_{\widetilde{U}_{i}} \widetilde{\rho}_{i} L\left(g, \widetilde{U}_{i}\right) .
$$

Then the V-signature theorem says that

$$
\operatorname{Sign}(Z)-\operatorname{Sign}^{V}(Z)=\operatorname{Sdef}(Z) .
$$

Kawasaki $[\mathrm{Ka}, \S 1]$ describes $\operatorname{Sdef}(Z)$ in the following global formulation. Here we also use the terminology of Chen-Ruan [CR, §3] at the same time.

Let $\Sigma Z$ be the singular locus of the orbifold $Z$, i.e., $\Sigma Z$ consists of the points $x \in Z$ whose isotropy groups $G_{x}$ are non-trivial. Since $G_{x}$ is abelian, the centralizer of each element of $G_{x}$ and the conjugacy class of $G_{x}$ are both isomorphic to $G_{x}$.

Let $\widetilde{\Sigma} Z$ be the twisted sector of $Z$ [CR, p. 7]. Namely, $\widetilde{\Sigma} Z$ consists of the pairs $\left\{(x, g) ; x \in \Sigma Z, g \in G_{x}, g \neq \mathrm{id}\right\}$ which has the orbifold structure as follows: Let $\left\{\widetilde{U}_{x} \rightarrow\right.$ $\left.U_{x}, G_{x}=\operatorname{Gal}\left(\widetilde{U}_{x} / U_{x}\right)\right\}$ be a local uniformazing system of $Z$ containing $x$, and let $\widetilde{U}_{x}^{g}$ be the fixed point locus of $g$ on $\widetilde{U_{x}}$. Since $G_{x}$ coincides with the centralizer of $g$ in $G_{x}, G_{x}$ itself acts on ${\widetilde{U_{x}}}^{g}$ possibly non-effectively in general. Then the systems $\left\{{\widetilde{U_{x}}}^{g} \rightarrow{\widetilde{U_{x}}}^{g} / G_{x}\right\}$ are patched globally and defines the orbifold chart on $\widetilde{\Sigma} Z$. Note that this orbifold structure need not be reduced. 
Let $\widetilde{\Sigma} Z_{k}(k=1, \ldots, \alpha)$ be the connected components of $\widetilde{\Sigma} Z$. For $(x, g) \in \widetilde{\Sigma} Z_{k}$, let $K_{x}$ be the kernel of the natural homomorphism from $G_{x}$ to the diffeomorphism group $\operatorname{Diffeo}\left({\widetilde{U_{x}}}^{g}\right)$ of ${\widetilde{U_{x}}}^{g}$. Then the cardinality $\left|K_{x}\right|$ is independent of the choice of $(x, g)$. We set $m_{k}=\left|K_{x}\right|$ and call it the multiplicity of the connected component $Z_{k}$ [Ka, p. 77]. If we change each local group from $G_{x}$ to $G_{x} / K_{x}$, then the system $\left\{{\widetilde{U_{x}}}^{g} \rightarrow{\widetilde{U_{x}}}^{g} /\left(G_{x} / K_{x}\right)\right\}$ defines a reduced orbifold structure on $Z_{k}$, which is written as $Z_{k}^{\text {red }}$.

Through the immersion $\widetilde{\Sigma} Z_{k}^{\text {red }} \longrightarrow Z$ defined by $(x, g) \mapsto x$, the orbifold normal bundle on $\widetilde{\Sigma} Z_{k}^{\text {red }}$ is well-defined. Then by patching the natural local equivariant L-forms, we can define the orbifold characteristic $\mathrm{L}^{\Sigma}$ on $\widetilde{\Sigma} Z_{k}^{\text {red }}$. We set

$$
\left\langle\mathrm{L}^{\Sigma}, \widetilde{\Sigma} Z_{k}\right\rangle=\frac{1}{m_{k}} \int_{\widetilde{\Sigma} Z_{k}^{\text {red }}}^{\text {orb }} \mathrm{L}^{\Sigma} \text {. }
$$

By [Ka, p. 78], we have

$$
\operatorname{Sdef}(Z)=\sum_{k=1}^{\alpha}\left\langle\mathrm{L}^{\Sigma}, \widetilde{\Sigma} Z_{k}\right\rangle .
$$

Next let $W$ be a simplicial toric variety. Then $W$ automatically has the structure of a complex orbifold. The following lemma seems to be well-known:

LEMMA 5.1.1. The $V$-signature $\operatorname{Sign}^{V}(W)$ coincides with the orbifold signature $\operatorname{Sign}^{\text {orb }}(W)$ defined in $\$ 3.1$.

PROOF. In Satake's sense, since the total V-Chern form of $W$ is generated by the first Chern form of V-line bundles of the torus invariant divisors, the V-signature is defined as the orbifold integral of the (Hirzebruch's L type) wedge product of these forms.

On the other hand, the Chow ring $A^{*}(W)$ is also generated by the torus invariant divisors and the intersection product of these divisors essentially comes from the homological intersection in the local uniformization systems.

In the local uniformization systems, since torus invariant divisors cross transversally with each other, the wedge product of the Chern forms and the intersection products of these divisors have the classical direct correspondence with natural compatibility (cf. [GH, Chap.0]). Therefore the assertion easily follows. We omit the details.

We go back to the situation in $§ 1.3$.

Proposition 5.1.2. For the compactified Fujiki-Oka modification $\bar{\mu}: \bar{Y} \rightarrow \bar{X}$, we have

$$
\operatorname{Sdef}(\bar{X})-\operatorname{Sdef}(\bar{Y})=\mathbf{s}\left(\frac{\mathbf{a}}{d}\right)-\sum_{i=1}^{n-1} \mathbf{s}\left(R_{i}\left(\frac{\mathbf{a}}{d}\right), N_{i}\left(\frac{\mathbf{a}}{d}\right)\right) .
$$

5.2. As a preparation for the proof of Proposition 5.1.2, we describe a connected component of the twisted sector of $Y$, and also give an explicit description of the characteristic $\mathrm{L}^{\Sigma}$ on it.

The fan $\widetilde{\sigma}$ in $\$ 1.3$ consists of the union of the faces of a nonsingular simplex $\sigma_{n}=$ $\mathbf{R}_{\geq 0} P_{1}+\cdots+\mathbf{R}_{\geq 0} P_{n-1}+\mathbf{R}_{\geq 0} Q$ and semi-unimodular simplexes $\sigma_{i}=\mathbf{R}_{\geq 0} P_{1}+\cdots+$ 
$\mathbf{R}_{\geq 0} P_{i-1}+\mathbf{R}_{\geq 0} Q+\mathbf{R}_{\geq 0} P_{i+1}+\cdots+\mathbf{R}_{\geq 0} P_{n}$ over $P_{n}(1 \leq i \leq n-1)$. The minimal orbifold chart of $Y=\mathrm{T}_{N} \mathrm{emb}(\Delta \widetilde{\sigma})$ is given by

$$
Y=\bigcup_{1 \leq i \leq n} U_{\sigma_{i}}, \quad\left\{\widetilde{U_{\sigma_{i}}} \stackrel{\pi_{i}}{\longrightarrow} U_{\sigma_{i}}, G_{i}=\operatorname{Gal}\left(\widetilde{U_{\sigma_{i}}} / U_{\sigma_{i}}\right)\right\} .
$$

Here $\pi_{n}$ is the identity map of $\widetilde{U_{\sigma_{n}}}=U_{\sigma_{n}}$ and the local uniformization map $\pi_{i}(1 \leq i \leq n-1)$ is the cyclic covering of order $a_{i}=\operatorname{mult}\left(\sigma_{i}\right)$ described as follows. Let $N_{i}$ be the sub-lattice of the original lattice $N$ generated by the primitive vectors $P_{1}, \ldots, P_{i-1}, Q, P_{i+1}, \ldots, P_{n}$. Then the natural equivariant morphism arising from $\left(N_{i}, \Delta_{\sigma}\right) \rightarrow\left(N, \Delta_{\sigma}\right)$

$$
\widetilde{U_{\sigma_{i}}}=\mathrm{T}_{N_{\mathrm{i}}} \mathrm{emb}\left(\Delta_{\sigma}\right) \simeq \mathbf{C}^{n} \longrightarrow U_{\sigma_{i}}=\mathrm{T}_{N} \mathrm{emb}\left(\Delta_{\sigma}\right)
$$

is nothing but the map $\pi_{i}$. The local Galois group $G_{i}$ is isomorphic to $N / N_{i}$, which is the cyclic group of order $a_{i}$.

We denote the torus invariant divisors on $\widetilde{U_{\sigma_{i}}}$ by

$$
D_{1, i}=\left.V\left(P_{1}\right)\right|_{\widetilde{U_{\sigma_{i}}}}, \ldots, D_{i, i}=\left.V(Q)\right|_{\widetilde{U_{\sigma_{i}}}}, \ldots, D_{n, i}=\left.V\left(P_{n}\right)\right|_{\widetilde{U_{\sigma_{i}}}} .
$$

Let $\left(z_{1}, \ldots, z_{n}\right)$ be the coordinate of $\widetilde{U_{\sigma_{i}}} \simeq \mathbf{C}^{n}$ so that $D_{k, i}$ is defined by $z_{k}=0(1 \leq k \leq n)$. We fix the generator $g_{i}$ of $G_{i} \simeq \mathbf{Z} / a_{i} \mathbf{Z}$. By Lemma 1.2.5, the proper fraction of $\sigma_{i}$ coincides with

$$
R_{i}(\mathbf{a} / d)=\frac{\left(\overline{a_{1}}, \ldots, \overline{a_{i-1}}, \overline{(-d)}, \overline{a_{i+1}}, \ldots, \overline{a_{n-1}}, 1\right)}{a_{i}} .
$$

We set

$$
a_{i, i}=-d, \quad a_{n, i}=1, \quad a_{k, i}=a_{k} \quad(1 \leq k \leq n-1, k \neq i) .
$$

By the same argument as in the proof of Proposition 1.2.3, the element $g_{i}^{j} \in G_{i}(0 \leq j \leq$ $a_{i}-1$ ) acts on $\widetilde{U_{\sigma_{i}}}$ as

$$
\left(z_{1}, \ldots, z_{n}\right) \longmapsto\left(\mathbf{e}\left(\frac{j a_{1, i}}{a_{i}}\right) z_{1}, \ldots, \mathbf{e}\left(\frac{j a_{n, i}}{a_{i}}\right) z_{n}\right)
$$

where $\mathbf{e}(x)=\exp (2 \pi \sqrt{-1} x)$. In the notation of (33), we define the subsets of I by

$$
\mathrm{I}_{j, i}^{0}=\mathrm{I}_{0}\left(j R_{i}(\mathbf{a} / d)\right), \quad \mathrm{I}_{j, i}^{\mathrm{ref}}=\mathrm{I}_{\mathrm{ref}}\left(j R_{i}(\mathbf{a} / d)\right), \quad \mathrm{I}_{j, i}^{*}=\mathrm{I}_{*}\left(j R_{i}(\mathbf{a} / d)\right) .
$$

Then the fixed point locus $V(j, i)=\left(\widetilde{U_{\sigma_{i}}}\right)^{g_{i}^{j}}$ of the action $g_{i}^{j}$ on $\widetilde{U_{\sigma_{i}}}$ coincides with the $\left|\mathrm{I}_{j, i}\right|$-dimensional torus invariant nonsingular subvariety

$$
V(j, i)=\bigcap_{k \in \mathrm{I} \backslash \mathbf{I}_{j, i}^{0}} D_{k, i}
$$

The tangent bundle and the normal bundle in $\widetilde{U_{\sigma_{i}}}$ of $V(j, i)$, respectively, have the natural direct sum decompositions

$$
T_{V(j, i)} \simeq \bigoplus_{k \in \mathrm{I}_{j, i}^{0}}\left[D_{k, i}\right], \quad N_{V(j, i) / \widetilde{U_{\sigma_{i}}}} \simeq \bigoplus_{k \in \mathrm{I} \backslash \mathrm{I}_{j, i}^{0}}\left[D_{k, i}\right]
$$


where $\left[D_{k, i}\right]$ is the line bundle which corresponds to the divisor $D_{k, i}$. The element $g_{i}^{j}$ acts on the direct factor $\left[D_{k, i}\right]\left(k \in \mathrm{I} \backslash \mathrm{I}_{j, i}^{0}\right)$ of the normal bundle with the eigenvalue $\mathbf{e}\left(j a_{k, i} / a_{i}\right)$. More precisely, if $k \in \mathrm{I}_{j, i}^{*}$, then $g_{i}^{j}$ acts $\left[D_{k, i}\right]$ as the rotation of angle $2 \pi j a_{k, i} / a_{i}$. If $k \in \mathrm{I}_{j, i}^{\mathrm{ref}}$, then $g_{i}^{j}$ acts on $\left[D_{k, i}\right]$ as the reflection.

By [AS, pp. 581-582], the equivariant L-form $L\left(g_{i}^{j}, \widetilde{U_{\sigma_{i}}}\right)$ is the differential form on $V(j, i)$ given by

$$
\begin{aligned}
L\left(g_{i}^{j}, \widetilde{U_{\sigma_{i}}}\right)= & \prod_{k \in \mathrm{I}_{j, i}^{*}} \operatorname{coth}\left(\frac{c_{1}\left(\left.\left[D_{k, i}\right]\right|_{V(j, i)}\right)}{2}+\frac{\sqrt{-1} \pi j a_{k, i}}{a_{i}}\right) \\
& \times \prod_{k \in \mathrm{I}_{j, i}^{0}} c_{1}\left(\left.\left[D_{k, i}\right]\right|_{V(j, i)}\right) \operatorname{coth} \frac{c_{1}\left(\left.\left[D_{k, i}\right]\right|_{V(j, i)}\right)}{2} \\
& \times \prod_{k \in \mathrm{I}_{j, i}^{\mathrm{ref}}} \tanh \frac{c_{1}\left(\left.\left[D_{k, i}\right]\right|_{V(j, i)}\right)}{2}
\end{aligned}
$$

where $c_{1}\left(\left.\left[D_{k, i}\right]\right|_{V(j, i)}\right)$ is the first Chern form of the restricted line bundle $\left.\left[D_{k, i}\right]\right|_{V(j, i)}$ and the product is the wedge product of the Chern forms.

As a preparation for the global formulation, we rewrite (48) in the following way. Since $\operatorname{gcd}\left(d, a_{i}\right)=1$ and $a_{n . i}=1$, the suffices $i$ and $n$ are not contained in $\mathrm{I}_{j, i}^{0}$. We put $r=\left|\mathrm{I}_{j, i}^{0}\right|$, and set

$$
\mathrm{I}_{j, i}^{0}=\left\{i_{1}, \ldots, i_{r}\right\} \subset I \backslash\{i, n\}, \quad I \backslash \mathrm{I}_{j, i}^{0}=\left\{i_{r+1}, \ldots, i_{n-r-2}\right\} \cup\{i, n\} .
$$

Then $j a_{i_{k}} \equiv 0\left(\bmod a_{i}\right)$ for $1 \leq k \leq r$, and $j a_{k} \not \equiv 0\left(\bmod a_{i}\right)$ for $k \in \mathrm{I} \backslash \mathrm{I}_{j, i}^{0}$. Moreover we have

$$
c:=\operatorname{gcd}\left(a_{i}, a_{i_{1}}, \ldots, a_{i_{r}}\right)>1 .
$$

We put $a_{i}^{\prime}=a_{i} / c$. There exists $j^{\prime}\left(1 \leq j^{\prime} \leq c-1\right)$ such that $j=a_{i}^{\prime} j^{\prime}$. Then (48) is rewritten as

$$
\begin{aligned}
L\left(g_{i}^{j}, \widetilde{U_{\sigma_{i}}}\right)= & \prod_{k \in \mathrm{I}_{j, i}^{*}} \operatorname{coth}\left(\frac{c_{1}\left(\left.\left[D_{k, i}\right]\right|_{V(j, i)}\right)}{2}+\frac{\sqrt{-1} \pi j^{\prime} a_{k, i}}{c}\right) \\
& \times \prod_{k \in \mathrm{I}_{j, i}^{0}} c_{1}\left(\left.\left[D_{k, i}\right]\right|_{V(j, i)}\right) \operatorname{coth} \frac{c_{1}\left(\left.\left[D_{k, i}\right]\right|_{V(j, i)}\right)}{2} \\
& \times \prod_{k \in \mathrm{I}_{j, i}^{\mathrm{ref}}} \tanh \frac{c_{1}\left(\left.\left[D_{k, i}\right]\right|_{V(j, i)}\right)}{2} .
\end{aligned}
$$

Note that (50) is symmetric with respect to the $r+1$ suffices $i, i_{1}, \ldots, i_{r}$.

Now the group $G_{i}$ acts on $V(j, i)$ possibly non-effectively. The kernel $K_{j, i}$ of the natural homomorphism $G_{i} \rightarrow \operatorname{Diffeo}(V(j, i))$ coincides with the subgroup $\left\langle g_{i}^{j}\right\rangle$ of $G_{i}$ generated by 
$g_{i}^{j}$. Therefore the chart

$$
\left\{V(j, i) \longrightarrow \pi_{i}(V(j, i)), \operatorname{Gal}\left(V(j, i) / \pi_{i}(V(j, i)) \simeq G_{i} / K_{j, i}\right\}\right.
$$

induces on $\pi_{i}(V(j, i))$ a reduced orbifold structure (cf. [CR, p. 3]) with $\left|G_{i} / K_{j, i}\right|=a_{i} / c$. By definition, the multiplicity $m_{j, i}$ of $V(j, i)$ coincides with

$$
m_{j, i}=\left|K_{j, i}\right|=c,
$$

which is also symmetric with respect to the suffices $i, i_{1}, \ldots, i_{r}$ by (49).

5.3. We globalize the observation in $\S 5$.2. We denote by $\overline{V(j, i)}$ the closure of $\pi_{i}\left(V_{j, i}\right)$ in $\bar{Y}$, which coincides with the $T_{N}$-invariant subvariety

$$
\overline{V(j, i)}=V\left(\sum_{\ell=r+1}^{n-r-2} \mathbf{R}_{\geq 0} P_{i_{\ell}}+\mathbf{R}_{\geq 0} Q+\mathbf{R}_{\geq 0} P_{n}\right) .
$$

As the restriction of $r+1$ local uniformazing systems of (44), we have the covering $\overline{V(j, i)}=$ $\sum_{\ell=0}^{r}\left(U_{i_{\ell}} \cap \overline{V(j, i)}\right)$ where we put $U_{i_{0}}=U_{i}$ and $U_{i_{\ell}}=U_{\sigma_{i_{\ell}}}$. This also induces the orbifold chart on $\overline{V(j, i)}$. More precisely, the system on $U_{i_{0}} \cap \overline{V(j, i)}=V(j, i)$ is given by (51) and the other systems $U_{i \ell} \cap \overline{V(j, i)}$ are essentially of the same type as this.

Then $\overline{V(j, i)}$ is one of the connected components of the twisted sector of $\bar{Y}$. The multiplicity $m_{j, i}$ of $\overline{V(j, i)}$ is already given by (52). The equivariant L-form on $\widetilde{U_{i_{\ell}}}(1 \leq \ell \leq r)$ which is patched together with (48) into the global form on $\overline{V(j, i)}$ is essentially of the same type as (48). The well-definedness of this globalization is clear from the symmetry property of (50) with respect to the $r+1$ suffices. We denote by $L^{\Sigma}\left(\overline{g_{i}^{j}}\right)$ this orbifold differential form on $\overline{V(j, i)}$. This is nothing but the restriction of $L^{\Sigma}$ to the component $\overline{V(j, i)}$.

LEMMA 5.3.1. We have an equality

$$
\begin{aligned}
\left\langle\mathrm{L}^{\Sigma}\left(\overline{g_{i}^{j}}\right)\right. & \overline{V(j, i)}\rangle \\
= & \frac{1}{a_{i}} \cdot \kappa_{n}\left\{\prod_{k \in \mathrm{I}_{j, i}^{*} \backslash\{i\}} \operatorname{coth}\left(\frac{\left[V\left(P_{k}\right)\right]}{2}+\frac{\sqrt{-1} \pi j a_{k}}{a_{i}}\right) \operatorname{coth}\left(\frac{[V(Q)]}{2}-\frac{\sqrt{-1} \pi j d}{a_{i}}\right)\right. \\
& \times \prod_{k \in \mathrm{I}_{j, i}^{0}}\left(\left[V\left(P_{k}\right)\right] \cdot \operatorname{coth} \frac{\left[V\left(P_{k}\right)\right]}{2}\right) \prod_{k \in \mathrm{I}_{j, i}^{\mathrm{ref}}} \tanh \frac{\left[V\left(P_{k}\right)\right]}{2} \\
& \left.\times\left(\prod_{k \in \mathrm{I}_{j, i}^{*} \backslash\{i\}}\left[V\left(P_{k}\right)\right]\right)[V(Q)]\right\}[\bar{Y}]
\end{aligned}
$$

where the product is the intersection product in the Chow ring $A_{\mathbf{Q}}^{*}(\bar{Y})$ and $\kappa_{n}$ is the degree $n$ part in the Taylor expansion evaluated at the fundamental class of $\bar{Y}$, which is the element in $H^{n}(\bar{Y}, \mathbf{Q}) \simeq \mathbf{Q}$.

PROOF. We denote by $\left(T_{\overline{V(j, i)}}\right)_{\mathbf{Q}},\left(N_{\overline{V(j, i)} / \bar{Y}}\right)_{\mathbf{Q}}$ the orbifold tangent bundle and the orbifold normal bundle in $Y$, respectively. The local decomposition (47) induces the global 
decomposition

$$
\left(T_{\overline{V(j, i)}}\right) \mathbf{Q} \simeq \bigoplus_{k \in \mathbf{I}_{j, i}^{0}}\left[V\left(P_{k}\right)\right], \quad\left(N_{\overline{V(j, i)} / \bar{Y}}\right)_{\mathbf{Q}} \simeq\left(\bigoplus_{k \in\left(I \backslash \mathbf{I}_{j, i}^{0}\right) \backslash\{i\}}\left[V\left(P_{k}\right)\right]\right) \bigoplus[V(Q)],
$$

where $\left[V\left(P_{k}\right)\right]$ is the $\mathbf{Q}$-line bundle (orbifold line bundle) associated to the $\mathbf{Q}$-divisor $V\left(P_{k}\right)$, etc. For each local system of $\overline{V(j, i)}$, say $V(j, i)=\overline{V(j, i)} \cap U_{i}$, we claim that

$$
\left.\frac{1}{m_{j . i}} \int_{V(j, i)} L^{\Sigma} \overline{\left(g_{i}^{j}\right)}\right|_{V(j, i)}=\frac{1}{a_{i}} \int_{V(j, i)} L\left(g_{i}^{j}, \widetilde{U}_{i}\right) .
$$

Indeed, the integral $\int_{V(j, i)} L\left(g_{i}^{j}, \widetilde{U}_{i}\right)$ is calculated on the non-reduced orbifold structure with the restriction to $V_{j, i}$ of the Galois group $G_{i}=\operatorname{Gal}\left(\widetilde{U}_{i} / U_{i}\right)$, it is $a_{i} / c$ times the reduced integral which is nothing but $\left.\int_{V(j, i)} L^{\Sigma}\left(\overline{g_{i}^{j}}\right)\right|_{V(j, i)}$ (cf. [CR, p. 5]). Since we have $m_{j . i}=c$ by (52), we have the claim (55).

By the same argument as in Lemma 5.1.1, the integral of the orbifold characteristic class is interpreted to be that on the rational Chow ring. Therefore the assertion follows from (45), (46), (50), (54) and (55).

Now we write down the explicit value $\left\langle\mathrm{L}^{\Sigma}\left(\overline{g_{i}^{j}}\right), \overline{V(j, i)}\right\rangle$ by using the terminology defined in $\$ 1$.

LEMMA 5.3.2. We have an equality

$$
\left\langle\mathrm{L}^{\Sigma} \overline{\left(g_{i}^{j}\right)}, \overline{V(j, i)}\right\rangle=\left(\left|\mathrm{I}_{j, i}^{0}\right|+1\right) \cot \left(j \cdot R_{i}\left(\frac{\mathbf{a}}{d}\right)\right)\left(N_{i}\left(\frac{\mathbf{a}}{d}\right)\right) .
$$

Proof. Since $[V(Q)]$ is contained in the product of the right-hand term of Lemma 5.3.1, by the same reason as in the proof of Proposition 3.2.1 and Remark 3.2.2, we may numerically assume that

$$
\begin{aligned}
{\left[V\left(P_{i}\right)\right] } & \equiv-\frac{a_{i}}{d}[V(Q)] \quad(1 \leq i \leq n-1), \\
{\left[V\left(P_{n}\right)\right] } & \equiv-\frac{1}{d}[V(Q)], \quad[V(Q)]^{n}=-\frac{d^{n-1}}{a_{1} \cdots a_{n-1}} .
\end{aligned}
$$

Therefore, it follows from Lemma 5.3.1 and (56) that

$$
\begin{aligned}
\left\langle\mathrm{L}^{\Sigma}\left(\overline{g_{i}^{j}}\right), \overline{V(j, i)}\right\rangle & =\frac{1}{a_{i}\left(\prod_{k \in \mathrm{I}_{j i}^{0}} a_{k}\right)} \cdot \kappa_{\left|\mathrm{I}_{j, i}^{0}\right|}\left\{\prod_{k \in \mathrm{I}_{j, i}^{*} \backslash\{i\}} \operatorname{coth}\left(\frac{a_{k} x}{2}+\frac{\sqrt{-1} \pi j a_{k}}{a_{i}}\right)\right. \\
& \left.\times \operatorname{coth}\left(\frac{-d x}{2}-\frac{\sqrt{-1} \pi j d}{a_{i}}\right) \prod_{k \in \mathrm{I}_{j, i}^{0}}\left(a_{k} x \cdot \operatorname{coth} \frac{a_{k} x}{2}\right) \prod_{k \in \mathrm{I}_{j, i}^{\mathrm{ref}}} \tanh \frac{a_{k} x}{2}\right\}
\end{aligned}
$$


where $\kappa_{\left|\mathrm{I}_{j, i}^{0}\right|}$ is the coefficient of the degree $\left|\mathrm{I}_{j, i}^{0}\right|$ term of Tayler expansion with respect to the variable $x$. The right-hand side of (57) coincides with

$$
\left(\left|\mathrm{I}_{j, i}^{0}\right|+1\right) \cot \left(j \cdot R_{i}\left(\frac{\mathbf{a}}{d}\right)\right)\left(N_{i}\left(\frac{\mathbf{a}}{d}\right)\right)
$$

by an easy calculation.

5.4 We prove Proposition 5.1.2. Since the restriction $\bar{Y} \backslash Y \longrightarrow \bar{X} \backslash X$ of $\bar{\mu}$ is an isomorphism, the contribution to the S-defects of both loci are cancelled. Since $X$ has an isolated cyclic orbipoint of type $\mathbf{a} / d$ such that the action (9) is small (i.e., any power of the map (9) is reflection free), we have

$$
\operatorname{Sdef}(X)=\mathbf{s}\left(\frac{\mathbf{a}}{d}\right)
$$

Now the set of the connected components of the twisted sector on $Y$ is written as

$$
\left\{\overline{V\left(j_{1}, i_{1}\right)}, \ldots, \overline{V\left(j_{\alpha}, i_{\alpha}\right)}\right\}
$$

for some $1 \leq i_{k} \leq n-1$ and some $1 \leq j \leq a_{i_{k}}(1 \leq k \leq \alpha-1)$. Since $\mathrm{L}^{\Sigma}=\bigoplus_{k=1}^{\alpha} \mathrm{L}^{\Sigma}\left(\overline{g_{i_{k}}^{j_{k}}}\right)$, it follows from (43), (58) that

$$
\left.\operatorname{Sdef}(\bar{X})-\operatorname{Sdef}(\bar{Y})=\mathbf{s}\left(\frac{\mathbf{a}}{d}\right)-\sum_{k=1}^{\alpha}\left\langle\mathrm{L}^{\Sigma} \overline{\left(g_{i_{k}}^{j_{k}}\right.}\right), \overline{V\left(j_{k}, i_{k}\right)}\right\rangle .
$$

Hence by Lemma 5.3.2, it suffices to prove

$$
\sum_{k=1}^{\alpha}\left(\left|\mathbf{I}_{j_{k}, i_{k}}^{0}\right|+1\right) \cot \left(j_{k} \cdot R_{i_{k}}\left(\frac{\mathbf{a}}{d}\right)\right)\left(N_{i_{k}}\left(\frac{\mathbf{a}}{d}\right)\right)=\sum_{i=1}^{n-1} \mathbf{s}\left(R_{i}\left(\frac{\mathbf{a}}{d}\right), N_{i}\left(\frac{\mathbf{a}}{d}\right)\right) .
$$

For any $(j, i)$ with $1 \leq i \leq n-1,1 \leq j \leq a_{i}-1$, the variety $\overline{V(j, i)}$ is one of the elements in the set (59). On the other hand, any element $\overline{V\left(j_{k}, i_{k}\right)}$ in the set (59) is counted $\left(\left|\mathrm{I}_{j_{k}, i_{k}}^{0}\right|+1\right)$ times if $(j, i)$ runs through all the pairs with $1 \leq i \leq n-1,1 \leq j \leq a_{i}-1$. From this, the assertion (60) follows by an easy calculation. Thus we complete the proof of Proposition 5.1.2.

5.5 The proof of Theorem 4.2.2. is straightforward from Corollary 2.1.2, Proposition 3.2.1, Lemma 5.1.1, Proposition 5.1.2, the formulas (42) and (1).

REMARK 5.5.1. With respect to related open questions, one may ask the following:

(1) What is the homogeneous version of (37) ? Namely can one drop the assumption that the last component of the numerator is 1 ?

(2) What is the reciprocity without the assumption of irreducibility of $\mathbf{a} / d$ ? This question is related to the computation problem of Hirzebruch-Zagier's Dedekind sum by iterated applications of reciprocities.

(3) Does the formula (37) relate to Beck reciprocity [B] ?

(4) What is the analogy for (37) of the relation between Dedekind sum and Dedekind eta function? 
(5) Can one apply the method of Fujiki-Oka modification to detect other types of reciprocity for Fourier-Dedekind sums (cf. [BR])?

(6) Do other types of quotient singularities or toric singularities have their "own reciprocities"?

Acknowledgement. The author thanks Professor Mutuo Oka for fruitful discussions and encouragements. Thanks are due to Professor Yoshinori Hamahata, Professor Miles Reid and also the referee for useful comments. Lastly he thanks Professor Tadao Oda for advices and encouragements.

\section{REFERENCES}

[A1] T. AshiKAGA, Local signature defect of fibered complex surfaces via monodromy and stable reduction, Comment. Math. Helv. 85 (2010), 417-461.

[A2] T. AshiKagA, Multidimensional continued fractions for cyclic quotient singularities and Dedekind sums, Preprint.

[AB] M. Atiyah And R. Bott, A Lefschetz fixed point formula for elliptic complexes II, Ann. of Math. 88 (1968), 451-491.

[AS] M. Atiyah And I. M. Singer, The index of elliptic operators III, Ann. of Math. 87 (1968), 546-604.

[BH1] A. Bayad And Y. Hamahata, Higher dimensional Dedekind sums in function fields, Acta. Arith. 152 (2012) no.1, 71-80.

[BH2] A. Bayad and Y. Hamahata, Multiple Dedekind-Rademacher sums in finite fields, Ramanujan J. 35 (2014), no. 3, 493-502.

[BR] A. BAYAD AND A. RaOuJ, Arithmetic of higher-dimensional Dedekind-Rademacher sums, J. Number Theory 132 (2012), 332-347.

[B] M. BECK, Dedekind cotangent sums, Acta Arith. 109 (2003), no. 2, 109-130.

[BR] M. BECK AND S. RoBINS, Computing the continuous discretely, Undergrad. Texts Math., Springer-Verlag, New York, 2007.

[BBD] A. A. Beilinson, J. Bernstein And P. Deligne, Faisceaux pervers, Analysis and topology on singular spaces, I (Luminy 1981), 5-171, Asterisque 100, Soc. Math. France, Paris, 1982.

[BRZ] A. Buckley, M. Reid AND S. Zhou, Orbifold Riemann-Roch and Hilbert series, Preprint.

[CR] W. Chen AND Y. RuAn, A new cohomology theory of orbifolds, Commun. Math. Phys. 248 (2004), 1-31.

[CLS] A. Cox, B. Little And H. Schenck, Toric varieties, Grad. Stud. Math. 124, American Mathematical Society, Providence, RI, 2011.

[D] V. I. Danilov, The geometry of toric varieties, Russian Math. Surveys 33 (1978), 97-154.

[Fuj] A. FuJIKI, On resolution of cyclic quotient singularities, Publ. Res. Inst. Math. Sci. 10 (1974/75), $293-328$.

[Fuk] Y. Fukumoto, The index of the $\operatorname{spin}^{c}$ Dirac operator on the weighted projective space and the reciprocity law of the Fourier -Dedekind sum, J. Math. Anal. Appl. 309 (2005), 674-685.

[FFU] Y. Fukumoto, M. Furuta AND M UE, $W$-invariants and Neumann-Siebenmann invariants for Seifert homology 3-sheres, Topology Appl. 116 (2001), 333-369.

[GH] P. GRIFFITHS AND J. HARRIS, Principles of Algebraic Geometry, John Wiley and Sons Inc., 1978.

[GM] M. GORESKY AND R. MACPHERSON, Intersection homology theory II, Invent. Math. 72 (1983), 77-129.

[GS] P. E. GUNNELlS AND R. SCZECH, Evaluation of Dedekind sums, Eisenstein cocycles, and special values of $L$-functions, Duke Math. J. 118 (2003), 229-260.

[HZ] F. HiRZEbruch AND D. B. Zagier, The Atiyah-Singer index theorem and elementary number theory, Publish or Perish, Wilmington, 1974.

[I1] M. N. IshidA, Torus embeddings and dualizing complexes, Tohoku Math. J. 32 (1980), 111-146.

[I2] M. N. IshidA, T-complexes and Ogata's zeta zero values, Adv. St. Pure Math. 15 (1989), 351-364.

[Ka] T. KAWASAKI, The signature theorem for $V$-manifolds, Topology 17 (1978), 75-83. 
[LR] N. C. LEUng And V. ReInER, The signature of a toric variety, Duke Math. J. 111 (2002), 253-286.

[MS] L. MAXIM AND J. SchÜRmAnn, Characteristic classes of toric varieties, Electron. Res. Announc. Math. Sci. 20 (2013), 109-120.

[Mc] P. MCMullen, On simple polytopes, Invent. Math. 113 (1993), 419-444.

[Od] T. OdA, Convex Bodies and Algebraic Geometry, An introduction to the theory of toric varieties, Translation from the Japanese, Ergeb. Math. Grenzgeb. (3) 15, Springer-Verlag, Berlin, 1988.

[Og1] S. OgAtA, Special values of zeta functions associated to cusp singularities, Tohoku Math. J. 37 (1985), 367-384.

[Og2] S. OGATA, Hirzebruch's conjecture on cusp singularities, Math. Ann. 296 (1993), 69-86.

[Ok] M. OKA, On the resolution of the hypersurface singularities, Adv. Stud. Pure Math. 8 (1986), 405-436.

[P] J. E. Pommersheim, Toric varieties, lattice points and Dedekind sums, Math. Ann. 295 (1993), 1-24.

[RG] H. Rademacher and E. Grosswald, Dedekind Sums, The Carus Mathematical Monographs, No. 16, The Mathematical Association of America, Washington, D.C., 1972.

[R] M. REID, Young person's guide to canonical singularities, in Algebraic Geometry Bowdoin 1985 (Brunswick, Maine, 1985), 345-414, Proc. Sympos. Pure Math. 46, Part 1, AMS, Providence, R.I., 1987.

[Sa] I. SATAKE, The Gauss-Bonnet theorem for $V$-manifolds, J. Math. Soc. Japan 9 (1957), 464-492.

[St] J. H. M. SteEnbrink, Mixed Hodge structure on the vanishing cohomology, Real and complex singularities (Proc. Ninth Nordic Summer School/NAVF Sympos. Math., Oslo, 1976), 525-563, Sijthoff and Noordhoff, Alphen aan den Rijn, 1977.

[Tsu] H. Tsuchinashi, Higher-dimensional analogues of periodic continued fractions and cusp singularities, Tohoku Math. J. 35 (1983), 607-639.

[U] G. URZÚA, Arrangements of curves and algebraic surfaces, J. Algebraic Geom. 19 (2010), 335-365.

[Z] D. ZAGIER, Higher dimensional Dedekind sum, Math. Ann. 202 (1973), 149-172.

FACULTY OF ENGINEERING

TOHOKU-GAKUIN UNIVERSITY

TAGAJO, MiYAGI, 985-8537

JAPAN

E-mail address: ashikaga@mail.tohoku-gakuin.ac.jp 